\title{
Implementation of a Body Force Model in OVERFLOW for Propulsor Simulations
}

\author{
H. Dogus Akaydin*, \\ Science and Technology Corporation, NASA Ames Research Center, Moffett Field, CA \\ Shishir A. Pandya ${ }^{\dagger}$, \\ NASA Ames Research Center, Moffett Field, CA
}

\begin{abstract}
We present an implementation of a propulsor model based on body force method into the Overflow computational fluid dynamics code to model turbofan engines and propulsors of similar type. The model estimates the forces imparted on the fluid by the blade camber surfaces as a body force source terms in the momentum and energy equations over grids that represent the rotor and stator of a fan stage. We tested the implementation on three cases: 1) Source Diagnostics Test (SDT) fan with R4 rotors, which has extensive test data on aerodynamic performance and rotor wake surveys, 2) A stand-alone Aeronaut TF8000 propulsor which is an off-the-shelf propulsor used on model aircraft and 3) The D8 aircraft model with TF8000 propulsors placed in a wind tunnel. Despite missing some of the features in the velocity profiles near the endwalls (i.e. hub and casing surfaces), the Overflow simulations with body force model predicted area-averaged flow speed and total pressure rise through the SDT propulsor within a few percent of the LDV (Laser Doppler Velocimetry) measurements. In the case of TF8000 propulsor on the D8 airframe, the model underpredicted mechanical power coefficient by several percent of the wind tunnel test results when the horizontal force balance condition over the airframe is targeted by tuning rotor speed. By investigating the upstream influence of the rotor swirl, it was found out that the induced swirl velocity effects upstream were relatively small and they rapidly vanished before reaching one fan diameter upstream of the fan face. The body force model provided insights on aerothermodynamics and aeromechanics of boundary layer ingesting propulsor; these insights could not be obtained by using the uniform pressure jump model.
\end{abstract}

\section{Introduction}

NASA's Advanced Air Transport Technology (AATT) project initiated an effort in 2008 to promote the development of advanced concepts and enabling technologies with aggressive targets to improve the fuel efficiency, noise and emissions of aircraft. One of the results of this effort was the D8 aircraft concept developed in partnership between Massachusetts Institute of Technology (MIT), Pratt \& Whitney, Aurora Flight Sciences, and NASA. ${ }^{1,2}$ This revolutionary concept is one of several being considered as a potential NASA Ultra Efficient Subsonic Transport (UEST) X-plane demonstrator.

The D8 aircraft concept has many key features including a double-bubble fuselage that produces more lift than a conventional one. Th D8 also features tightly-integrated propulsors that benefit from Boundary Layer Ingestion (BLI) as seen on Figure 1. The propulsors are integrated such that the lower portion of the nacelles is the upper surface of the rear fuselage and the outer sides of the nacelle are a part of the vertical stabilizers. This integration of the propulsors with the airframe allows the propulsors to ingest a large portion of the fuselage boundary layer. While in a conventional configuration, this boundary layer results in a momentum deficit (or loss of kinetic energy), the D8 propulsors ingest the boundary layer reducing the resultant kinetic energy loss. An additional consequence of the slower flow ingested by the propulsors is a

\footnotetext{
*Research Scientist, Computational Aerosciences Branch, NAS Division, AIAA Member

${ }^{\dagger}$ Aerospace Engineer, Computational Aerosciences Branch, NAS Division, AIAA Senior Member

This material is declared a work of the U.S. Government and is not subject to copyright protection in the United States.2017
} 
lower engine exhaust velocity. This also results in a reduced kinetic energy loss. Thus, the D8 configuration has a much higher aerodynamic efficiency compared to conventional aircraft. ${ }^{2-4}$

A repercussion of the boundary layer ingestion (BLI) is that the flow entering the propulsor is no longer uniform; it varies both in radial and circumferential directions at the inlet. As traditional numerical methods for modeling airflow through a fan assume uniform incoming flow, accurate computational assessment of the performance gains due to BLI require a propulsor model which can more accurately account for the distortions in the flow entering the engine.

To date, a series of high-fidelity CFD (computational fluid dynamics) simulations of the D8 aircraft using the Overflow code has been performed. ${ }^{4}$ These simulations relied on a simple actuator disk boundary condition, which imposes a uniform static pressure jump across the propulsor, to model the D8 propulsors (Figure 1). In agreement with the corresponding wind tunnel experiments, these simulations quantified the increase in the aerodynamic efficiency due to boundary layer ingestion. ${ }^{4}$ However, the uniform pressure jump model currently available in Overflow has some inherent deficiencies. First, the uniform pressure jump model requires an empirical input, i.e. the amount of pressure jump, either supplied from experimental data or found by trial and error until an imposed condition is met. Simulations hence cannot predict the airframe performance based solely on the fan blade geometry and rotation speed. This not only allows making predictions based on a given fan blade but also on variations of it, which may not readily have any test data, for design and optimization studies. Second, since a constant static pressure jump is imposed, the uniform pressure jump model cannot fully respond to the local (radial, circumferential and streamwise) variations in the flow field. Therefore the effect of inflow velocity distortions on aerodynamic performance may not be predicted accurately. Third, the uniform pressure jump does not create or negate swirl which may become important if stators needs to be placed in the solution to increase force balance accuracy. Modeling swirl may also be important for propulsors that ingest distorted flow and that may have their swirl fields upstream and downstream may interact, especially if their fans are co-rotating and closely-packed like in the D8 aircraft. Fourth, mere application of a pressure jump results in generation of a large amount of spurious entropy generation across the location it is applied. At transonic flight conditions that require relatively high pressure jumps, lack of swirl, generation of spurious entropy and inherent unsteadiness of the flow may make the steady-state RANS flow simulations numerically challenging Hence, there appeared a need for a better propulsor model which can avoid imposing any empirical inputs, have a physics-based interaction with local flow conditions, account for swirl and be numerically stable even at relatively high-thrust situations. While it is possible to incorporate spinning propulsor blades into the simulations, such high-fidelity and time-accurate propulsor models would make the overall simulation tasks for the development of this aircraft prohibitively - and possibly unnecessarily - expensive. As a result, we sought an advanced propulsor model which can fit into the steady-state RANS simulation framework that focuses more on external aerodynamics of the aircraft and does not create an excessive computational burden.

Numerous works in the literature present various implementations of actuator disks, actuator lines or blade elements. A list of notable ones include the works by O'Brien and Smith, ${ }^{5}$ Fejtek and Roberts, ${ }^{6}$ Zori and Rajagopalan, ${ }^{7}$ and Chaffin and Berry ${ }^{8}$ a survey of various actuator disk models are given in. ${ }^{9}$ These models were primarily developed for rotorcraft and wind turbine applications. Two variants of the model by Chaffin and Berry ${ }^{8}$ are readily available in Overflow ${ }^{10}$ as "prop/rotor model" and are widely used in rotorcraft simulations. Few actuator disk models were developed specifically for turbomachinery applications, such as the works by Joo and Hynes, ${ }^{11}$ and Kim et al. ${ }^{12}$

Apart from the actuator disk or similar approaches, a source term based method specifically for turbomachines was introduced by Marble ${ }^{13}$ and utilized by Gong et al. ${ }^{14}$ and others. ${ }^{15,16}$ In these methods, the effects of the rotor and stator blades on the fluid (flow turning, pressure rise and enthalpy rise) are expressed as body forces which are pre-computed from single-passage CFD simulations that involve only a pair of rotor blades and a pair of stator blades. These body forces are then introduced as source terms to the systems of equations solved for in 3D throughflow simulations of the full propulsor. A model that was recently presented by Hall et al. ${ }^{17,18}$ takes this approach a step further by computing the source terms during the 3D throughflow simulation itself by using a blade loading model. This model represents the rotor or stator blades as 2D camber surfaces, which is a more realistic approach than actuator disk or blade element models in terms of geometric representation and a less demanding approach than full-wheel 3D-blade simulations in terms of grid generation and flow solution expense. The model does not impose values on flow field variables but adds source terms which themselves are determined from blade rotation speed, blade camber representation and local velocity vector. As a result, the model is able to interact with the flow field while representing the blade 
geometry and does not require any empirical inputs such as pressure rise or enthalpy rise. Since the model acts over a 3D volume rather than a 2D disk, its interaction with the flow field takes place gradually over a volume representing the blade row, rather than discontinuously through a surface in the flow field. While the blade loading is modeled by inviscid, incompressible flow assumptions, the source terms are added both in the momentum and energy equations in an aerothermodynamically consistent way so as to avoid generation of spurious entropy in compressible flow situations. Finally, this model does not need aerodynamic lookup tables since the blade loading is computed at runtime with a relatively small overhead.

Implementing various propulsor models and testing them on multiple cases would be an ideal and a major undertaking. Due to its aforementioned advantages in modeling fidelity and implementation, and to our ongoing collaboration with its developers, we currently proceeded with implementing only the model by Hall et al. ${ }^{17}$ into the Overflow code.

The first goal of this paper is to compare our previous propulsor modeling approach, which relied on the uniform pressure jump model available in Overflow, with our recent approach which uses the body force method that we implemented. The second goal is to sufficiently document this implementation. A detailed description of the model by Hall et al. ${ }^{17}$ and its implementation Overflow code is given in the Methods section. The model is tested in cases which simulate a relatively high-thrust propulsor, a relatively lowthrust propulsor and finally a propulsor integrated in D8 airframe with boundary layer ingestion. Further descriptions and results of these test cases are shown in Test Cases section. Finally, an overall discussion of the model and of its results are provided in the Summary section.

\section{Methods}

\section{II.A. The Uniform Pressure Jump Model}

The uniform pressure jump model is the traditional propulsor modeling approach in OVERFLOW. This model increases the static pressure by an amount $\Delta p$ through a surface subset of the grid as

$$
\begin{aligned}
p_{J} & =p_{\text {avg }}-0.5 \Delta p \\
p_{J+1} & =p_{\text {avg }}+0.5 \Delta p
\end{aligned}
$$

where $J$ is the selected grid index in the streamwise direction, $p_{\text {avg }}=0.5\left(p_{J}+p_{J+1}\right)$ is the average pressure before the application of the actuator disk boundary condition and $\Delta p$ is the thrust force per actuator disk area. The pressure jump is applied as a boundary condition, not a source term, at a constant $J$ plane and $\Delta p$ is constant for all $K$ and $L$ indices on which the boundary condition is applied. There are no changes imposed on any other flow variable and there is no modeling of swirl or torque. This is the model known as the "Actuator disk" boundary condition in the Overflow code, version 2.21. ${ }^{10}$ Since there is a variety of actuator disk models with various levels of sophistication in the literature, this model is referred to as "uniform pressure jump model" in this text to eliminate any possible confusion with other models.

\section{II.B. The Body Force Model}

The body force model described here was recently presented by by Hall et al. ${ }^{17}$ This model estimates the local momentum and energy imparted by the rotor and stator blades onto the fluid by a thin airfoil approximation and adds them as source terms to the right-hand-side of the inviscid, steady-state momentum equation (Eqn. 2b) and energy equation (Eqn. 2c):

$$
\begin{aligned}
\nabla \cdot(\rho \mathbf{V}) & =0 \\
\mathbf{V} \cdot \nabla \mathbf{V}+\frac{\nabla p}{\rho} & =\mathbf{f} \\
\mathbf{V} \cdot \nabla h_{t} & =\mathbf{V} \cdot \mathbf{f}+\dot{e}
\end{aligned}
$$

where $\mathbf{V}, \rho, p$, and $h_{t}$ are respectively the velocity, density, static pressure and total enthalpy of the fluid; $\mathbf{U}$ is the velocity of a point on the camber surface representation of the blade, $\mathbf{f}$ is the body force per unit volume of fluid at that location and $\dot{e}$ is the rate of entropy per unit volume of fluid under that body force. The source term component $\dot{e}$ corresponds to entropy generation which can be derived from the differential form of Gibbs equation

$$
T \nabla s=\nabla h-\frac{1}{\rho} \nabla p
$$


along with Eqns. $2 \mathrm{~b}$ and $2 \mathrm{c}$ as

$$
\dot{e}=T \cdot \mathbf{V} \nabla s
$$

The differential form of Euler turbine equation, on the other hand, states that

$$
\mathbf{V} \cdot \nabla h_{t}=\Omega r f_{\theta}
$$

where $\Omega$ is angular velocity of the blade row, $r$ is the distance of the point from the rotation axis and $f_{\theta}$ is the component of the body force (f) tangential to the rotation direction. The angular velocity of the blade row is the rotation speed of the rotor in radians per second and is zero for stator blades. Combining Eqns. $2 \mathrm{c}, 4$ and 5 it can be shown that

$$
\dot{e}=T \cdot \mathbf{V} \nabla s=-\mathbf{W} \cdot \mathbf{f}
$$

where $\mathbf{W}=\mathbf{V}-\mathbf{U}=\mathbf{V}-\Omega r \boldsymbol{\Theta}$ is the velocity of the flow relative to the blade and $\boldsymbol{\Theta}$ is a unit vector pointing in the direction of the tangential velocity.

The signed magnitude of the body force $\mathbf{f}$ is modeled as

$$
f=\frac{2 \pi \delta\left(\frac{1}{2}|\mathbf{W}|^{2}\right)}{\frac{2 \pi r}{B}\left|n_{\theta}\right|}
$$

which approximates the local thrust of a low-solidity, thin-airfoil propeller. In this expression, $B$ is the number of blades and $n_{\theta}$ is the circumferential component of $\mathbf{n}$, the unit vector normal to the camber surface of the blade at that point. The value of $f$ can be positive or negative depending on $\delta$, which is the local deviation angle of the flow from the surface tangent in the flow direction (Figure 2), which is given by

$$
\delta=\arcsin \left(\frac{\mathbf{W} \cdot \mathbf{n}}{|\mathbf{W}| \cdot|\mathbf{n}|}\right)
$$

The direction of $\mathbf{f}$ is determined by enforcing the following constraints on it:

$$
\begin{aligned}
\mathbf{W} \cdot \mathbf{f} & =0 \\
(\mathbf{W} \times \mathbf{n}) \cdot \mathbf{f} & =0 \\
\operatorname{sign}(\mathbf{f} \cdot \mathbf{n}) & =-\operatorname{sign}(\mathbf{W} \cdot \mathbf{n})
\end{aligned}
$$

The first constraint comes from the isentropic flow turning assumption that requires force to be normal to the streamlines and effectively makes $\dot{e}=0$ and reduces Eqn. 2c to 5

$$
\mathbf{V} \cdot \nabla h_{t}=\Omega r f_{\theta}
$$

The second constraint limits $\mathbf{f}$ to lie on the plane shared by $\mathbf{W}$ and $\mathbf{n}$. Finally, the third constraint sets the direction of $\mathbf{f}$ such that the body force reduces the flow deviation angle $\delta$.

Details of an algorithmic way of computing $\mathbf{f}$ will be provided in the next section along with the particular implementation of the body force model into the Overflow code.

\section{II.C. Computational Method and Implementation}

In this work, we carried out the flow simulations using Overflow, ${ }^{10}$ which is an implicit, Reynolds-Averaged Navier-Stokes (RANS) solver developed by NASA for high-fidelity CFD simulations using curvilinear structured overset grids. We generated the overset grids using Chimera Grid Tools (CGT), which is a NASAdeveloped toolset to generate body-fitted structured overset grid systems. ${ }^{19,20}$ The overset grid connectivity was accomplished by using DCF (Domain Connectivity Function) routines internal to the Overflow solver. ${ }^{21}$ In the current implementation, the grid zone where the body force model is applied is generated as follows:

1. Generate a structured grid definition of the camber surface of the rotor blade. In this work, we first create a structured surface grid of the rotor blade and extracted the camber surface simply by taking the average of the coordinates of corresponding points on the suction and pressure sides at each radial section (Figure 3a,b). Orient the blade in a propulsor Cartesian frame of reference $X Y Z$ such that the axis of the propulsor is aligned with the $X$-axis. 
2. At each vertex $P_{\mathrm{c}}(X, Y, Z)$ on the camber surface grid, compute the axisymmetric coordinates $P_{\mathrm{c}}(X, r, \theta)$ :

$$
\begin{aligned}
& r_{\mathrm{c}}=\sqrt{Y_{\mathrm{c}}^{2}+Z_{\mathrm{c}}^{2}} \\
& \theta_{\mathrm{c}}=\arctan \left(Z_{\mathrm{c}} / Y_{\mathrm{c}}\right)
\end{aligned}
$$

3. At each vertex $P_{\mathrm{c}}$ on the camber surface grid find the surface normal $\mathbf{n}_{\mathrm{c}}=\left\langle n_{\mathrm{c}, X}, n_{\mathrm{c}, Y}, n_{\mathrm{c}, Z}\right\rangle$ and compute (Figure 3f):

$$
\begin{aligned}
& n_{\mathrm{c}, r}=\left(X_{\mathrm{c}} n_{\mathrm{c}, Y}+Z_{\mathrm{c}} n_{\mathrm{c}, Z}\right) / r_{\mathrm{c}} \\
& n_{\mathrm{c}, \theta}=\left(-Z_{\mathrm{c}} / n_{\mathrm{c}, Y}+Y_{\mathrm{c}} n_{\mathrm{c}, Z}\right) / r_{\mathrm{c}}
\end{aligned}
$$

The surface normals of the camber surface should point in the positive rotation direction around the $X$-axis of the propulsor; this can be accomplished by multiplying $\mathbf{n}_{\mathrm{c}}$ by -1 if necessary. In this implementation, we computed the surface normals using a routine readily available in CGT.

4. Flatten the camber surface representation on $X Y$ plane such that each vertex $P_{\mathrm{c}}(X, Y, Z)$ on the camber surface corresponds to a point $P_{X Y}(x, y=r, 0)$ on the flattened camber surface representation (Figure 3c). Accordingly, rotate the actual camber surface normals $\left(n_{c}\right)$ to find the corresponding camber surface normals on the flattened camber surface grid $\left(n_{X Y}\right)$ :

$$
\begin{aligned}
& n_{X Y, X}=n_{\mathrm{c}, X} \\
& n_{X Y, Y}=n_{\mathrm{c}, r} \\
& n_{X Y, Z}=n_{\mathrm{c}, \theta}
\end{aligned}
$$

5. Extend the flattened grid upstream and downstream by a few (e.g. 4 to 10) grid lines to ensure proper overlap with neighboring grids (Figure 3d).

6. Fully revolve the flattened camber grid around the $X$-axis to generate a structured volume grid of revolution by computing

$$
\begin{aligned}
X & =X_{\mathrm{c}} \\
Y & =r_{\mathrm{c}} \cos (K \Delta \theta) \\
Z & =r_{\mathrm{c}} \sin (K \Delta \theta)
\end{aligned}
$$

for each point $P(X, Y, Z)$ in the rotor grid zone (Figure $3 \mathrm{e}$ ). Here $K=1,2,3, \ldots, K_{\max }$ is the index number of the point in the periodic direction of the grid and $\Delta \theta=2 \pi /\left(K_{\max }-1\right)$. In the grid system of this work, we used $K_{\max }=181$ ( $K=1$ plane overlapping with $K=181$ plane) which resulted in $\Delta \theta=2^{\circ}$.

Additionally, revolve the camber surface orientation metrics for the subzone where the body force model will be applied (Figure 3f).

$$
\begin{aligned}
n_{X} & =n_{\mathrm{c}, X} \\
n_{Y} & =n_{\mathrm{c}, r} \cos (K \Delta \theta)-n_{\mathrm{c}, \theta} \sin (K \Delta \theta) \\
n_{Z} & =n_{\mathrm{c}, r} \sin (K \Delta \theta)+n_{\mathrm{c}, \theta} \cos (K \Delta \theta) \\
n_{\theta} & =n_{\mathrm{c}, \theta} \\
\Theta_{X} & =0 \\
\Theta_{Y} & =0 \\
\Theta_{Z} & =1
\end{aligned}
$$

where $\Theta_{X}, \Theta_{Y}, \Theta_{Z}$ are the components of $\Theta$ the unit vector in the direction of the tangential velocity of the rotor blade.

The body force model is applied to the subzone $\left(J_{\mathrm{b}}:-J_{\mathrm{e}}, 1: K_{\max }, L_{\mathrm{b}}:-L_{\mathrm{e}}\right)$ where $J_{\mathrm{b}}$ and $J_{\mathrm{e}}$ are the number of added grid lines to extend the grid in upstream and downstream directions in Step 5. $L_{\mathrm{b}}$ and $L_{\mathrm{e}}$ are the number of grid lines retracted from the hub and casing surfaces, respectively, to 
constrain the application of the body force model in radial direction. In case of the stator grid zone, a radial retraction of at least one grid line is necessary to avoid division-by-zero error while computing the source terms at $L=1$ and $L=L_{\max }$ since $W$ is zero due to static wall boundary conditions applied at the hub and the casing surfaces. In case of the rotor grid zone, no body force zone retraction is necessary at tip $\left(L_{\mathrm{e}}=1\right)$ if there is already a physical gap between the rotor tip and the casing. In addition, no body force retraction is necessary at the hub $\left(L_{\mathrm{b}}=1\right)$ since $W$ is never zero due to rotation.

7. Finally, if the axis of revolution ( $X$-axis) of the installed propulsor grid is not aligned with the $x$-axis of the solution domain (i.e. non-zero toe and/or pitch angles), then rotate the propulsor grids as well as the source term orientation metrics accordingly (Figure $3 \mathrm{~g}$ ). In this implementation we expressed the orientation of the propulsor as a rotation by an angle $\phi$ around a unit orientation vector $\mathbf{a}=\left\langle a_{x}, a_{y}, a_{z}\right\rangle$ and used Rodrigues' Rotation Formula ${ }^{22}$ to rotate the orientation metrics:

$$
\begin{aligned}
\mathbf{n}_{x y z} & =\mathbf{R n}_{X Y Z}^{T} \\
\boldsymbol{\Theta}_{x y z} & =\mathbf{R} \Theta_{X Y Z}^{T}
\end{aligned}
$$

where

$$
\mathbf{R}=\mathbf{I}+\sin (\phi) \mathbf{A}+(1-\cos (\phi)) \mathbf{A}^{2}
$$

and where

$$
\begin{gathered}
\mathbf{A}=\left[\begin{array}{ccc}
0 & -a_{z} & a_{y} \\
a_{z} & 0 & -a_{x} \\
-a_{y} & a_{x} & 0
\end{array}\right] \\
\mathbf{I}=\left[\begin{array}{lll}
1 & 0 & 0 \\
0 & 1 & 0 \\
0 & 0 & 1
\end{array}\right]
\end{gathered}
$$

8. Write $r, n_{x}, n_{y}, n_{z}, n_{\theta}, \Theta_{x}, \Theta_{y}, \Theta_{z}$ in a file to be read by Overflow for computation of the source terms during the flow solution.

The extraction, flattening, revolution and rotation of the camber surface and computation of its orientation metrics are programmed in executable codes that are called from CGT grid scripts with appropriate user inputs.

A set of subroutines added to the Overflow code reads the camber surface orientation metrics files for the rotor and the stator grids and computes the source terms at each body force grid point at each flow solver iteration as follows:

1. Compute the components of relative velocity $\mathbf{W}=\mathbf{U}-\Omega r \Theta$ in the Cartesian frame of the flow simulation domain:

$$
\begin{aligned}
& W_{x}=U_{x}-\Omega r \Theta_{x} \\
& W_{y}=U_{y}-\Omega r \Theta_{y} \\
& W_{z}=U_{z}-\Omega r \Theta_{z}
\end{aligned}
$$

where $U_{x}, U_{y}, U_{z}$ and $W_{x}, W_{y}, W_{z}$ are the components of $\mathbf{\Theta}, \mathbf{U}, \mathbf{W}$ in the cartesian frame of reference of the flow solution domain.

2. Compute the normal component of $\mathbf{W}$

$$
W_{n}=\mathbf{W} \cdot \mathbf{n}=W_{x} n_{x}+W_{y} n_{y}+W_{z} n_{z}
$$

Note that $W_{n}$ can have a positive or negative value.

3. Compute the local deviation angle:

$$
\delta=\arcsin \left(W_{n} /|W|\right)
$$

where $|\mathbf{W}|=\sqrt{W_{x}^{2}+W_{y}^{2}+W_{z}^{2}}$ 
4. Compute the signed magnitude of the force according to the following blade loading model:

$$
f=\frac{B|\mathbf{W}|^{2} \delta}{2 r\left|n_{\theta}\right|}
$$

Note that both $\delta$ and $f$ must have the same sign as $W_{n}$, as stipulated by the third constraint in Eqns. 9

5. Compute the components of $\mathbf{W}_{n}=W_{n} \mathbf{n}$, which is the projection of $\mathbf{W}$ onto $\mathbf{n}$ :

$$
\begin{aligned}
& W_{n, x}=W_{n} n_{x} \\
& W_{n, y}=W_{n} n_{y} \\
& W_{n, z}=W_{n} n_{z}
\end{aligned}
$$

6. Compute the components of $\mathbf{W}_{t}=\mathbf{W}-\mathbf{W}_{n}$ :

$$
\begin{aligned}
& W_{t, x}=W_{x}-W_{n, x} \\
& W_{t, y}=W_{y}-W_{n, y} \\
& W_{t, z}=W_{z}-W_{n, z}
\end{aligned}
$$

7. Compute the components of $\mathbf{t}=\mathbf{W}_{t} /\left|\mathbf{W}_{t}\right|$, which is the blade-tangent vector aligned with the local relative velocity:

$$
\begin{aligned}
t_{x} & =W_{t, x} /\left|W_{t}\right| \\
t_{y} & =W_{t, y} /\left|W_{t}\right| \\
t_{z} & =W_{t, z} /\left|W_{t}\right|
\end{aligned}
$$

where $\left|W_{t}\right|=\sqrt{W_{t, x}^{2}+W_{t, y}^{2}+W_{t, z}^{2}}$.

8. Compute the components of $\mathbf{f}$ normal and tangential to the blade surface:

$$
\begin{gathered}
f_{n}=-f\left|W_{t}\right| /|W| \\
f_{t}=f\left|W_{n}\right| /|W|
\end{gathered}
$$

9. Compute the components of $\mathbf{f}=f_{n} \mathbf{n}+f_{t} \mathbf{t}$ :

$$
\begin{aligned}
& f_{x}=f_{n} n_{x}+f_{t} t_{x} \\
& f_{y}=f_{n} n_{y}+f_{t} t_{y} \\
& f_{z}=f_{n} n_{z}+f_{t} t_{z}
\end{aligned}
$$

The body force routines in Overflow add the source terms contributions to the residuals as follows:

$$
\begin{aligned}
& S_{x}=S_{x}-f_{x} \rho \mathcal{J}^{-1} \\
& S_{y}=S_{y}-f_{y} \rho \mathcal{J}^{-1} \\
& S_{z}=S_{z}-f_{z} \rho \mathcal{J}^{-1} \\
& S_{e}=S_{e}-s_{e} \mathcal{J}^{-1}
\end{aligned}
$$

where $s_{e}=\Omega r\left(f_{x} \Theta_{x}+f_{y} \Theta_{y}+f_{z} \Theta_{z}\right)$ and $\mathcal{J}$ is the metric Jacobian of the curvilinear grid at that vertex. 


\section{Test Cases and Results}

In this section we present the three cases on which we tested the body force model:

1. A stand-alone Source Diagnostics Test (SDT) propulsor with R4 rotor blades

2. A stand-alone Aeronaut TF8000 propulsor

3. The D8 aircraft with TF8000 propulsors

The SDT propulsor with R4 rotor blades is chosen as a benchmark case due to availability of experimental aerodynamic data obtained in NASA Glenn Research Center's $9 \times 15 \mathrm{ft}$ wind tunnel during the Source Diagnostics Test campaign. ${ }^{23-26}$ The rotor wake survey data will be used to validate the body force model implementation in this work. The SDT case also serves as a relatively high-thrust propulsor case to test the applicability of the body force and uniform pressure jump models.

Aeronaut TF8000 is a commercial ducted fan used on the wind tunnel model of the D8 aircraft. ${ }^{3}$ The stand-alone TF8000 propulsor case corresponds to the podded (non-BLI) variant of the D8 aircraft where propulsors are placed away from the fuselage and hence ingest undistorted flow. This case serves as a relatively low-thrust application for the body-force model. In addition, rotor and stator blade counts of TF8000 propulsor are much fewer than those of the SDT propulsor (5 and 4, respectively, versus 22 and 26); therefore, the TF8000 propulsor is also a case to test the applicability of the body model for propulsors with few blade counts.

The D8 aircraft case has extensive experimental rotor speed and force balance data as well as detailed CFD data with uniform pressure jump model for both the original D8 configuration with BLI and the podded variant of it without BLI. ${ }^{3,4}$ While the data from the podded case complements the stand-alone TF 8000 simulations, the data from the integrated case will be used to assess the predictions of the body force model subject to boundary layer ingestion. Therefore this case is the ultimate test case to demonstrate the benefits of the body force model implemented in this work.

\section{III.A. Source Diagnostics Test (SDT) propulsor with R4 rotor blades}

In late 2002 Envia et al. at NASA Glenn Research Center completed a series of wind tunnel tests on a singlestage bypass flow model of a modern turbofan engine. ${ }^{23}$ In this test campaign known as Source Diagnostics Test (SDT), they collected and published a large amount of aeroacoustic and aerodynamic diagnostics data including wake surveys between the rotor and the stator. ${ }^{24-26}$ In this section we compare the predictions of the body force model with the aerodynamic data obtained in the SDT campaign. The SDT fan model comprises of a hub, a nacelle, an "R4 rotor" of 22 blades and a "low-noise stator" of 26 swept-back stator blades, as shown in Figure 4a. The hub and the interior of the nacelle (i.e. "casing") are the versions designed specifically for the low-noise stator as noted in the data package documentation. Note that the hub geometry, which was originally truncated in the downstream end, is extended with a smoothed tapering cone in this CFD validation model in order to alleviate vortex shedding that may hinder steady-state solution convergence.

Sideview of the curvilinear structured overset grid system to simulate this geometry is shown in Figure 4b-e. The origin of the simulation domain is at the center of the inlet face of the model. The domain is extended about 35 times the length of the model in each spatial direction via multi-level cartesian off-body grids, which are automatically generated by the Overflow solver. All near-body grids were generated with a nominal maximum stretching ratio of 1.1 along all $(\mathrm{J}, \mathrm{K}$ and $\mathrm{L})$ grid directions. Double-fringe overset connectivity with no orphan points was achieved with a donor quality factor of 0.9 or better. The total number of grid points in the solution domain is about 35 million, 25 million of which constitute the 7 nearbody grids covering the model. With a wall spacing of $1.27 \times 10^{-6} \mathrm{~m}$, the near-body grids have a $y^{+}$value of less than and close to one over the interior surfaces of the propulsor and less than two over most of the other wall surfaces.

For obtaining the steady-state results presented in this work we used a Beam-Warming scheme ${ }^{27}$ with constant maximum CFL number (ramped from 5 to 10) and central-differencing with matrix dissipation (ramped from DIS2 $=5$ and DIS4=0.1 to DIS2=2 and DIS4=0.04). Turbulence was modeled using the Spalart-Allmaras (SA) model. ${ }^{28}$ Freestream conditions were set to $\mathrm{Re}_{\infty}=2.31$ million per meter, $\mathrm{M}_{\infty}=0.1^{\mathrm{a}}$

${ }^{\text {a }}$ The wind tunnel tests were actually conducted at $\mathrm{M}_{\infty}=0.05$ instead, with a bell-mouth mounted to create engine inlet conditions that would be obtained at $\mathrm{M}_{\infty}=0.1$, which is the freestream speed the test article was designed for. 
and $\mathrm{T}_{\infty}=288 \mathrm{~K}$. Spinner boundary condition at the rotor speed was applied to the segment of the hub surface that interfaces with the rotor grid.

During the SDT campaign, an extensive set of unsteady LDV (Laser Doppler Anemometry) data was collected at two immersion stations in the wake of the rotor at various engine speeds. ${ }^{25}$ The highest and the lowest engine speeds with LDV data available were 12,657 rpm (100\%, "design speed" for take-off) and 7808 rpm (61.7\%, "approach speed"). In this work, we simulated both of these engine speeds using the body force method as well as the uniform pressure jump model currently available in the Overflow code. The residuals of the simulations with the body force model dropped than seven orders of magnitude within 40,000 iterations, which took eight hours of wall-clock time using 128 Haswell cores of Pleiades supercomputer at NASA Ames Research Center. Aerodynamic force convergence was achieved within the first 20,000 iterations. The simulations with uniform pressure jump model, however, did not converge to a steady-state solution despite higher artificial dissipation coefficients, lower CFL numbers and various other solver parameter combinations tried for extended wall-clock times. In these uniform pressure jump simulations of the SDT geometry, residuals would first drop a few orders of magnitude, then increase to a level less than the initial order of magnitude and remain oscillating around that level. Although the flow field within the propulsor would look fairly settled, oscillations along the free shear layer of the exhaust jet further downstream would prevent the solution from converging to a steady-state solution. Despite this lack of convergence, however, the results of uniform pressure jump model simulations at approach speed are included here for a qualitative demonstration along with the predictions of the body force model developed in this work.

The contour plots of various quantities throughout the fan are shown in Figure 5. In the case of the body force model simulation, which is shown in the left column, rotor loads kinetic energy into the fluid by swirling as well as accelerating it downstream (Figure 5a, b). Static pressure of the fluid also rises significantly through the rotor region. The stator converts the swirl velocity almost completely to additional static pressure and streamwise velocity (Figure 5a,b,f). As a result, the total pressure increases rapidly within the rotor region due to the added energy while it remains approximately constant through the stator region which ideally does not add or remove any energy. (Figure 5d) Since the local blade loading forces are set up to be normal to the streamlines, they turn the flow isentropically and produce a negligible amount of entropy through the rotor or stator zones (Figure 5e).

In the case of uniform pressure jump model simulation, which is present in the right column, the variation of flow quantities are driven only by a static pressure jump which is constant both in the radial and circumferencial directions and imposed as a step function from a constant streamwise grid index to the next streamwise grid index, as described in Eqns. 1a. As a result, the modeling fidelity and response observed in the body force model solutions are not observed in the uniform pressure jump model solution. (Figure 5f).

Figure 6 shows the line plots of streamwise and swirl velocity components at two stations between the rotor and the stator as predicted by body force model simulations along with the corresponding LDV data taken during the SDT campaign. The body force model overpredicted the streamwise velocities, especially near the hub.

In $7,808 \mathrm{rpm}$ case, the body force model predicted the velocity profiles at both stations reasonably well except near the endwalls (hub and casing surfaces). In 12,657 rpm case, the streamwise velocity profile predictions are good except near the hub surface at both stations. However, the swirl velocity predictions in this higher-speed case miss the speedup in swirl near the casing while overpredicting the swirl near the hub. Deviations from the measurements near the endwalls are not too surprising since the model assumes that the blades are infinitely long and it does not account for any finite span and secondary flow effects near the hub and casing ends of the blades. Furthermore, the magnitude and the direction of blade loading $f$ is based on inviscid and incompressible flow assumptions while the flow modeled here is viscous and compressible. Nevertheless, the current body force model predicted area-averaged flow speeds with good accuracy. For example, in the case of $12,657 \mathrm{rpm}$, the area-averaged streamwise and swirl velocity components computed from the SDT data in Figure 6 at Station 1 are $171 \mathrm{~m} / \mathrm{s}$ and $138 \mathrm{~m} / \mathrm{s}$, respectively. At Station 2, the measured values of these velocity components were $177 \mathrm{~m} / \mathrm{s}$ and $136 \mathrm{~m} / \mathrm{s}$. The body force model predicted these quantities as $172 \mathrm{~m} / \mathrm{s}$ and $133 \mathrm{~m} / \mathrm{s}$ at Station 1 and $176 \mathrm{~m} / \mathrm{s}$ and $127 \mathrm{~m} / \mathrm{s}$ at Station 2. Therefore, both velocity components were predicted with less than $5 \%$ error at both stations. The same conclusion is true for the lower speed simulations at $7808 \mathrm{rpm}$. The total pressure ratios for $12,657 \mathrm{rpm}$ and 7,808 rpm were measured by Hughes et al. ${ }^{26}$ as 1.509 and 1.159, respectively. The body force method simulations at these speeds predict the respective total pressure ratios as 1.491 and 1.181 , which are less than $2 \%$ different. Note that these predictions with the body force model are accomplished without utilizing any empirical input (e.g. 
$\Delta p=$ thrust force per actuator disk area) as in the uniform pressure jump model; therefore, the predictive capability of the body force model even without any corrections is remarkable.

\section{III.B. TF8000 Propulsor}

The TF8000 propulsors were used during a test of the D8 model in the 14x22 wind tunnel of NASA Langley Research Center. ${ }^{3}$ In the D8 model, two TF8000 propulsors were tightly integrated into the fuselage and the vertical tail junction area so that the engines could ingest a part of the boundary layer that develops over the fuselage (Figure 1). In order to assess the effects of boundary layer ingestion, a "podded" variant of the D8 was also tested as a control article. In the podded variant, the propulsors were mounted away from the fuselage to avoid boundary layer ingestion. For both configurations the force balance measurements were carried out at several fan rotation speeds to measure the resulting horizontal (streamwise) force $C_{x}$ at each fan rotation speed and to determine the rotation speed at which the horizontal force nearly vanishes. It was found that the horizontal force balance would be achieved at approximately 11,100 rpm when the propulsors were integrated (BLI case) and at approximately 11,550 rpm when the propulsor were podded (non-BLI case). A set of earlier CFD simulations demonstrated that the inlet conditions of the podded propulsors were nearly the same as those of a stand-alone propulsor in freestream. Characteristics of standalone TF8000 propulsors are therefore representative of those of the podded D8 propulsors at 11,550 rpm rotation speed.

The overall geometry of a stand-alone TF8000 propulsor is shown in Figure 7a. The TF8000 propulsor has fewer rotor and stator blades than the SDT fan; however, the chords of TF8000 blades are relatively longer, especially in case of the stator blades.

Overset grid generation and flow simulations of the TF8000 fan were carried out in a similar way to the SDT fan simulations using the Beam-Warming scheme with constant maximum CFL number ramped from 15 to 5 and matrix dissipation ramped from DIS2 $=5$ and DIS $4=0.1$ to DIS $2=2$, DIS $4=0.04$. The freestream conditions were set to $\mathrm{Re}_{\infty}=1.86$ million per meter, $\mathrm{M}_{\infty}=0.088$ and $\mathrm{T}_{\infty}=288 \mathrm{~K}$, which correspond to the conditions at the beginning of the test section of the wind tunnel. With the same wall spacing as in the SDT case $\left(1.27 \times 10^{-} 6 \mathrm{~m}\right)$, the near-body grids had a $y^{+}$value of less than and close to one over the interior surfaces of the propulsor and less than 1.3 over the rest of the wall surfaces. The overset grid system for the TF8000 fan has around 18 million grid points, around 15 million of which belong to the 7 near-body grids covering the propulsor. For both models (uniform pressure jump and body force models) the residual convergence with more than eight orders of magnitude was achieved within 20,000 iterations, which took about four hours of wall-clock time on 128 Haswell cores. Steady-state aerodynamic force convergence was achieved for both models within the first 10,000 iterations.

Predictions of the integrated quantities obtained by the two models are shown in Table 1. The power input from the propulsor to the flow is measured by the mechanical power coefficient $C_{\mathrm{PK}}$,

$$
C_{\mathrm{PK}}=\frac{\int_{f a n}\left(p_{t, \infty}-p_{t}\right)(\mathbf{V} \cdot \mathbf{n}) d A}{q_{\infty} V_{\infty} S_{r e f}}
$$

where $p_{t, \infty}$ and $p_{t}$ are freestream and local values of total pressure, $V_{\infty}$ is freestream velocity, $q_{\infty}=$ $0.5 \rho_{\infty} V_{\infty}^{2}$ is freestream dynamic pressure and $S_{r e f}$ is the reference area used for normalizing D8 wind tunnel model aerodynamic coefficients. The integration region fan is formally a closed control volume that is within the propulsor and encloses both the rotor and the stator. Since $\mathbf{V}$ across the inner walls of (hub and casing) of the propulsor is zero, the integration region reduces to two planar surfaces covering inlet and outlet of the propulsor as shown in Figure 9d by solid black lines. The integral is computed by carrying out the integration on the inlet surface and on the outlet surface separately, and then summing the two integration results.

Table 1. Mechanical power coefficient and mass flow rates predicted by the two models compared with values measured during the wind tunnel experiments. Experimental values are inferred from. ${ }^{3,29,30} C_{\dot{m}}=\dot{m} /\left(S_{\text {ref }} M_{\infty}\right)$

\begin{tabular}{l|ll}
\hline Method & $C_{P K}$ & $C_{\dot{m}}$ \\
\hline Experiment & $0.049 \pm 0.001$ & $0.0275 \pm 0.0006$ \\
Body Force & 0.046 & 0.0288 \\
Uniform Pressure Jump & 0.048 & 0.0292 \\
\hline
\end{tabular}


The body force model underpredicted the mechanical power coefficient by $6 \%$ while overpredicting the mass flow coefficient by $5 \%$. While this difference may be due to the body force model itself, it may also be due to any uncharacterized inconsistencies between the particular ways the experimental and the CFD data were collected and postprocessed as well as any uncertainties in matching the horizontal force. The impact of horizontal force setting on $C_{P K}$ values will be addressed further on the next test case.

Contour plots of the quantities extracted from the converged solutions are presented in Figure 8. The boundaries of the rotor and stator blade zones are drawn by black lines and the location of the uniform pressure jump condition is evident on the $C_{p}$ contour in the right column. Total pressure and Mach number has more radial variation in case of the body force model than in the uniform pressure jump model. The variations of static pressure in radial and streamwise directions within the rotor zone are also remarkably different; the body force model is able to capture the gradual transition from low-pressure near leading edge to high-pressure near the trailing edge of the rotor zone as well as the radial load distribution weighed towards the tip rather than the root. A small amount of entropy is generated in a region within the rotor tip gap in case of the body force model, in addition to the entropy generated within the viscous boundary layers over the hub and nacelle surfaces. The uniform pressure jump model, however, generates a large amount of entropy as in the case of SDT fan simulations.

\section{III.C. The D8 aircraft in LaRC $14 \times 22$ wind tunnel}

The D8 simulations were carried out by incorporating the TF8000 grids to the D8 grid system generated for prior work ${ }^{4}$ in conjunction with the wind tunnel experiments carried out by Uranga et al. ${ }^{3}$ in the $14 \times 22$ wind tunnel at NASA Langley Research Center. The domain for the D8 simulations extends from the beginning of the contraction section of the wind tunnel to the end of the diffuser section and is centered at the nose of the model. Only the starboard half of the model and the wind tunnel were simulated under the assumption that the flow is symmetric on either half of the model. The walls of the wind tunnel were modeled as adiabatic slip-walls and the mid-plane ( $y=0$ plane) of the wind tunnel was given symmetry boundary conditions. The streamwise direction of the wind tunnel was aligned with the positive $x$ axis. The tunnel flow was driven by back pressure boundary condition at the end of the diffuser section until the Mach number at the beginning of the test section matches that computed from the conditions measured during the wind tunnel tests.

The grid system has about 162 million vertices spread over 70 grid zones. The wakes of the wings, fuselage, horizontal and vertical tail as well as the plume of the propulsor are captured using dedicated wake grids for those components. The $y^{+}$values are around one over almost all of the wall surfaces. The rotor speed that brings the net horizontal force coefficient $\left(C_{x}\right)$ (including drag and thrust) to nearly zero $\left(C_{x}<<C_{D}\right)$ is determined to be $11,100 \pm 10 \mathrm{rpm}$ during the wind tunnel tests. This rotational speed was used in the body force model simulations. In the uniform pressure jump simulations, the pressure jump was adjusted until the horizontal force integrated over all the wall surfaces of the D8 model plus the inlet and exit planes of the propulsor is nearly zero. The numerical scheme and solver parameters are the same as the standalone TF8000 case except that the solution was started by a series of grid sequencing solutions up to two levels of grid coarsening to accelerate the start-up of the tunnel flow. These initial runs were carried out with low-Mach preconditioning turned on $(\mathrm{BIMIN}=0.1)$, matrix dissipation coefficients increased (DIS2 $=5.0$ and DIS4=0.1) and maximum CFL number reduced (ramped from 0.5 to 1.0). Once the flow settled, multigrid process and low-Mach preconditioning were turned off, dissipation coefficients were ramped down to DIS2 $=2.0$ and DIS4 $=0.04$, and the maximum CFL number was ramped up to 1.5. After a converged solution with the uniform pressure jump boundary condition is obtained, that solution was used to start the simulation with the body force model. The simulations were performed using 800 Haswel cores on the Pleiades supercomputer. Tunnel start-up were given about 205,000 grid sequencing iterations (which took 7.5 hours of wall-clock time) followed by 10,000 iterations with multigrid (which took 3.5 hours of wall-clock time). Final convergence in force history using the uniform pressure jump model required about 155,000 non-multigrid iterations which took 26 more hours of wall-clock time and included a few attempts to tune the pressure jump. Final convergence in force history using the body force model took about 100,000 iterations which took about 18 hours. The body force simulation required more iterations to converge to a steady-state solution and took about $3 \%$ longer to complete the same number of iterations; however, it did not require additional runs for tuning any parameter to match the experiments. Although we have not yet conducted a rigorous uncertainty quantification study, earlier grid refinement studies, solver parameter tests and comparisons with experimental data showed that this grid system and selection of solver parameters have enough precision to resolve the effects of minute configuration changes on aerodynamic coefficients. ${ }^{4,31}$ 
Table 2. Aerodynamic forces predicted by the models and measured with the wind tunnel experiments. Experimental values are inferred from. ${ }^{3,29,30} C_{\dot{m}}=\dot{m} /\left(S_{\text {ref }} M_{\infty}\right)$

\begin{tabular}{l|llll}
\hline Method & $C_{x}$ & $C_{z}$ & $C_{\mathrm{PK}}$ & $C_{\dot{m}}$ \\
\hline Experiment & $0.0000 \pm 0.0006$ & $0.644 \pm 0.001$ & $0.045 \pm 0.001$ & $0.0267 \pm 0.0006$ \\
Uniform Pressure Jump & 0.0002 & 0.651 & 0.045 & 0.0282 \\
Body Force, 11,100 rpm & 0.0028 & 0.672 & 0.039 & 0.0275 \\
Body Force, 11,450 rpm & 0.0005 & 0.678 & 0.043 & 0.0281 \\
\hline
\end{tabular}

The force and power coefficients obtained with the two models are compared with the experimental values in Table. 2 The first row comes from the data published on the D8 wind tunnel tests. The second row is obtained by tuning the uniform pressure jump to produce a horizontal force coefficient which is nearly zero. The third row is obtained by using the body force model with the rotor speed that produced zero horizontal force during the wind tunnel experiments $(11,100 \mathrm{rpm})$. This row demonstrates the direct predictive capability of the body force model in that it relies only on the specified design data (i.e. the rotor camber shape and an externally specified rotor speed). In this case, the power coefficient of the propulsor is underpredicted by about $13 \%$, resulting in a horizontal force coefficient of $C_{x}=0.0028$. In order to resolve the effect of the horizontal force balance on $C_{P K}$ values, we tuned the rotor speed used in body force model to bring $C_{x}$ closer to zero. This resulted in a rotor speed of 11,450 rpm, with a small amount of $C_{x}$ remaining, and a $C_{P K}$ value which is about $4 \%$ less than the experimental one at $C_{x}=0$. From this, we can conclude that $13 \%$ error in prediction of $C_{P K}$ includes a $9 \%$ difference due to a mismatch of the horizontal force coefficients, which may be due not only to the propulsor model itself but also other possible sources of uncertainty throughout the experimental and computational processes. Once the body force model is tuned to create a $C_{x}$ value closer to the experimental one, the difference between the $C_{P K}$ values is reduced to $4 \%$.

Contour plots of Mach number and total pressure coefficients extracted from the solutions are presented in Figure 9. The velocity fields within the propulsors are noticeably different, both among the two models for a given region and among the parts of the flow above and below the hub for a given propulsor (9a). Through a given part of the flow, especially through the one above the hub, the body force model accelerates the flow near the core more than it does the near the endwall. The uniform pressure jump model, on the other hand, seems to accelerate the flow more uniformly across a given part. Both models predict a generally higher-speed flow in the part of the flow above the hub (undistorted) than that below the hub; which agrees with the distorted inflow condition that the flow is readily faster at the part of the fan face above the hub than the fan face below the hub. However, the speed difference between the parts above or below the hub seems to be smaller in the case of the body force model than it is in the case of the uniform pressure jump model. It appears that the fan blades attenuate the inflow distortions by mixing the fluid through the swirl they introduce, as observed previously by others. ${ }^{17}$

According to Figure 9b, the uniform pressure jump method suggests that the blade loading is more uniform from hub to casing and the blades are loaded more in the part above the hub than the part below the hub. In contrast, the body force model predicts that the blades are loaded more near the casing than near the hub and that the blades are loaded more in the part below the hub than in the part above the hub.

The attenuation effect of the fan blades is also evident in Figure 9c. While the form of the ingested boundary layer is preserved through the uniform pressure jump fan, the body force model redistributes the incoming total pressure and increases the total pressure more uniformly between the parts above and below the hub. The difference between the two total pressure fields is made more clear by subtracting the field predicted by the uniform pressure jump model from that predicted by the body force model, as shown in 9d. It is clear that the body force model predicts more mechanical work input in the casing region of the lower part of the and less work input most other regions, except for the core region of the part above the hub. Note that despite these profound differences in variations of flow field parameters between the two models, the $C_{P_{K}}$ values they predict are relatively close.

The effect of the propulsor model selection on total pressure distribution across the fan face plane was not noticeable. However, some changes in the streamlines and velocity components were observed between the uniform pressure jump model and the body force model. As seen on the lower row of Figure 10a, the swirl velocity generated by the body force model bends the streamtraces slightly inboard (towards $y=0$ plane) as they approach the fan face. The induced curvature is more pronounced near the fuselage wall 
where the flow loses momentum and becomes more prone to the induced swirl. The downstream effect of swirl is to focus the plume, which otherwise tends to spread outboard in an extension of the inflow direction. The upstream velocity components are only slightly modified by the body force method. At about the fan face $(x=2.79 \mathrm{~m})$, the body force method predicts a few percent lower streamwise $V_{x}$ velocity in the upper flow passage and a similarly higher $V_{x}$ velocity in lower flow passage, as compared to the uniform pressure jump model. The increase in $V_{y}$ at that station is relatively more pronounced, especially near the tip and the lower side of the hub. Note that the influence of the fan rotation on the velocity field does not reach more than a fan diameter upstream, as evidenced by the total overlap of the velocity profiles predicted by the two fan models at $x=2.67 \mathrm{~m}$ station.

\section{Summary}

Boundary Layer Ingestion (BLI) is a common feature of transport aircraft proposed for NASA's call for fuel-efficient aircraft. Accurate and affordable modeling of the interaction of the fan blades with inflow distortions requires medium-fidelity engine models that can respond to local flow field and are applicable to the thrust conditions needed by flow simulations of full-scale airframe at transonic speeds. Such a model, which adds source-terms to the Navier-Stokes equations based on an estimation of forces imparted on the fluid by the blade camber surfaces, was recently presented by Hall et al. ${ }^{17}$ In this work, we implemented this body force model into the Overflow solver and developed a framework to generate the grids and input files necessary for using this model in Overflow solver. We then tested our implementation on three cases: 1) SDT fan, which is a relatively high-thrust case, 2) Aeronaut TF8000 propulsor, which is a relatively low-thrust case and 3) Integrated D8 configuration, which is a boundary layer ingestion case.

In case of the SDT fan, the body force model missed some of the velocity profile features near the endwalls, especially in the high-speed case, probably due to compressibility and blade blockage effects. However, it predicted mass flow rates, total pressure ratios and average streamwise and swirl velocities within a few percent of the wind tunnel results. The uniform pressure jump model, on the other hand, could not produce a converged steady-state RANS solution for SDT fan at the conditions tested.

In the cases of TF 8000 propulsor, the body force model underpredicted the propulsive power by $6 \%$ in the stand-alone case and by $13 \%$ in the D8-mounted case when the rotor speed was set directly to the value which created $C_{x}=0$ in the wind tunnel experiments. A majority of the underprediction in the D8-mounted case was in conjunction with not achieving the horizontal force equilibrium at the given rotor speed. When the rotor speed used in the body force model is tuned to nearly achieve a horizontal force balance as in the experiments, the underprediction in $C_{P K}$ was reduced to $4 \%$ from $13 \%$. The additional fidelity that comes with the body force model provided valuable insights on how the mechanical work input takes place within a boundary-layer-ingesting propulsor and how the propulsor affects the velocity profiles upstream and downstream.

In the future work, we will implement corrections to the body force model to address endwall, compressibility, low blade count and viscosity effects to improve the predictions for high-thrust fans as well as low blade-count fans. We will also address any possible inconsistencies between the experimental and computational data processing which might, in part, have led to underprediction of mechanical power coefficient of TF8000 propulsor. Another future work item is to implement actuator disk models of various fidelity in Overflow and compare them with the body force model implementation described in this work, with the goal of determining the level of fidelity necessary for BLI propulsor modeling.

\section{Acknowledgements}

Dr. David K. Hall of the MIT Gas Turbine Laboratory provided a description of the source term computation algorithm given in Section II.C. Dr. Edmane Envia of NASA Glenn Research Center provided the SDT aerodynamic data and geometry definition files. NASA Advanced Air Transport Technology (AATT) project provided the funding for this work. NASA Advanced Supercomputing (NAS) Division at NASA Ames Research Center provided computing resources.

\section{References}

\footnotetext{
${ }^{1}$ Greitzer, E. M., Bonnefoy, P. A., la Rosa Blanco, E. D., Dorbian, C. S., Drela, M., Hall, D. K., Hansman, R. J., Hileman, J. I., Liebeck, R. H., Lovegren, J., Mody, P., Pertuze, J. A., Sato, S., Spakovszky, Z. S., Tan, C. S., Hollman, J. S., Duda, J. E., Fitzgerald, N., Houghton, J., Kerrebrock, J. L., Kiwada, G. F., Kordonowy, D., Parrish, J. C., Tylko, J., Wen, E. A., and Lord,
} 
W. K., "N+3 Aircraft Concept Designs and Trade Studies, Final Report, Volume I," Contractor Report CR-2010-216794/VOL1, NASA, 2010.

${ }^{2}$ Drela, M., "Development of the D8 Transport Configuration," 29th Applied Aerodynamics Conference, Honolulu, HI, June 27-30 2011, AIAA 2011-3970.

${ }^{3}$ Uranga, A., Drela, M., Greitzer, E. M., Titchener, N. A., Lieu, M. K., Siu, N. M., and Huang, A. C., "Preliminary Experimental Assesment of the Boundary Layer Ingestion Benefit for the D8 Aircr aft," 52nd Aerospace Sciences Meeting, National Harbor, MD, January 13-17 2014, AIAA 2014-0906.

${ }^{4}$ Pandya, S. A., Huang, A., Espitia, A., and Uranga, A., "Computational Assesment of the Boundary Layer Ingesting Nacelle Desing of the D8 Aircraft," 52nd Aerospace Sciences Meeting, National Harbor, MD, January 13-17 2014, AIAA 2014-0907.

${ }^{5}$ O'Brien, D. M. and Smith, M. J., "Analysis of Rotor-Fuselage Interactions Using Various Rotor Models," 43rd AIAA Aerospaces Sciences Meeeting and Exhibit, Reno, NV, January 10-13 2005, AIAA 2005-468.

${ }^{6}$ Fejtek, I. and Roberts, L., "Navier-Stokes computation of wing/rotor interaction for a tilt rotor in hover," AIAA Journal, Vol. 30, No. 11, 1992, pp. 2595-2603.

${ }^{7}$ Zori, L. A. and Rajagopalan, R. G., "NavierStokes Calculations of RotorAirframe Interaction in Forward Flight," Journal of the American Helicopter Society, Vol. 40, No. 2, 1995, pp. 57-67.

${ }^{8}$ Chaffin, M. S. and Berry, J. D., "Helicopter Fuselage Aerodynamics under a Rotor by Navier-Stokes Simulation," Journal of the American Helicopter Society, Vol. 42, No. 3, 1997, pp. 235-243.

${ }^{9}$ Chuiton, F. L., "Actuator Disc Modelling for Helicopter Rotors," Aerospace Science and Technology, Vol. 8, 2004, pp. 285-297.

${ }^{10}$ Nichols, R. H. and Buning, P. G., "Users Manual for OVERFLOW 2.2," Technical report, NASA, 2010.

${ }^{11}$ Joo, W. G. and Hynes, T. P., "The Simulation of Turbomachinery Blade Rows in Asymmetric Flow Using Actuator Disks," ASME Journal of Turbomachinery, Vol. 119, No. 4, 1997, pp. 723-732.

${ }^{12}$ Kim, S., Yang, S., Lee, D., Baftalovski, S., and Makarov, V., "Three-dimensional flow calculation around/through isolated nacelle with an actuator disk modeling," 35th AIAA/ASME/SAE/ASEE Joint Propulsion Conference and Exhibit, June 1999, AIAA 66-2668.

${ }^{13}$ Marble, F. E., "Three-dimensional flow in turbomachines," Aerodynamics of Turbines and Compressors, edited by W. R. Hawthorne, chap. C, Princeton University Press, Princeton, New Jersey, 1964, pp. 83-166.

${ }^{14}$ Gong, Y., Tan, C. S., Gordon, K. A., and Greitzer, E. M., "A Computational Model for Short-Wavelength Stall Inception and Development in Multistage Compressors," ASME Journal of Turbomachinery, Vol. 121, No. 4, 1998, pp. $726-734$.

${ }^{15}$ Defoe, J. J. and Spakovszky, Z. S., "Effects of Boundary-Layer Ingestion on the Aero-Acoustics of Transonic Fan Rotors," ASME Journal of Turbomachinery, Vol. 135, No. 5, 2013.

${ }^{16}$ Andreas Peters, Zoltn S. Spakovszky, W. K. L. and Rose, B., "Ultrashort Nacelles for Low Fan Pressure Ratio Propulsors," ASME Journal of Turbomachinery, Vol. 137, No. 2, 2014.

${ }^{17}$ Hall, D. K., Greitzer, E. M., and Tan, C. S., "Analysis of Fan Stage Conceptual Design Attributes for Boundary Layer Ingestion," Journal of Turbomachinery, Vol. 139, 2017, pp. 0710121-0710129.

${ }^{18}$ Hall, D. K., Analysis of civil aircraft propulsors with boundary layer ingestion, Ph.D. thesis, Massachusetts Institute of Technology, 2015.

${ }^{19}$ Chan, W., Gomez, R., Rogers, S., and Buning, P., "Best Practices in Overset Grid Generation," 32nd AIAA Fluid Dynamics Conference and Exhibit, St. Louis, Missouri, Jun 24-26 2002, AIAA-2002-3191.

${ }^{20}$ Chan, W., "Developments in Strategies and Software Tools for Overset Structured Grid Generation and Connectivity," 20th AIAA Computational Fluid Dynamics Conference and Exhibit, Honolulu, Hawai, Jun 27-30 2011, AIAA-2011-3051.

${ }^{21}$ Chiu, I. T. and Meakin, R., "On Automating Domain Connectivity for Overset Grids," 33rd Aerospace Sciences Meeting and Exhibit, January 9 -12 1995, Reno, NV.

${ }^{22}$ Mason, M. T., Mechanics of Robot Manipulation, MIT Press, 2001.

${ }^{23}$ Envia, E., "Fan Noise Source Diagnostic Test Completed and Documented," Technical Memorandum TM-2003-211990, NASA, 2003.

${ }^{24}$ Hughes, C. E., "Aerodynamic Performance of Scale-Model Turbofan Outlet Guide Vanes Designed for Low Noise," 40th AIAA Aerospaces Sciences Meeeting and Exhibit, Reno, NV, January 14-17 2002, AIAA 2002-374.

${ }^{25}$ Podboy, G. G., Krupar, M. J., Hughes, C. E., and Woodward, R. P., "Fan Noise Source Diagnostic Test — LDV Measured Flow Field Results," Technical Memorandum TM-2003-212330, NASA, 2003.

${ }^{26}$ Hughes, C. E., Jeracki, R. J., Woodward, R. P., and Miller, C. J., "Fan Noise Source Diagnostic Test — RotorAlone Aerodynamic Performance Results," Technical Memorandum TM-2005-211681, NASA, 2005.

${ }^{27}$ Pulliam, T. H. and Chaussee, D. S., "A diagonal form of an implicit approximate-factorization algorithm," Journal of Computational Physics, Vol. 39, No. 2, 1981, pp. 347-363.

${ }^{28}$ Aupoix, B. and Spalart, P. R., "Extensions of the Spalart-Allmaras Turbulence Model to Account for Wall Roughness," International Journal of Heat and Fluid Flow, Vol. 24, 2003, pp. 454-462.

${ }^{29}$ Casses, C. J., Aerodynamic Benefits of Boundary Layer Ingestion for the D8 Double-Bubble Aircraft, Ph.D. thesis, Massachusetts Institute of Technology, 2013.

${ }^{30} \mathrm{Lieu}, \mathrm{M} . \mathrm{K}$., Quantification of the Boundary Layer Ingestion Benefit for the D8-Series Aircraft Using a Pressure Rake System, Ph.D. thesis, Massachusetts Institute of Technology, 2015.

${ }^{31}$ Pandya, S. A., "External Aerodynamics Simulations for the MIT D8 "Double-Bubble" Aircraft Design," 7th International Conference on Computational Fluid Dynamics, Big Island, HI, July 9-13 2012, AIAA7-4304. 
(a)

D8 aircraft

$1: 11$ scale wind tunnel model

$S_{\text {ref }}=1.088 \mathrm{~m}^{2}$

Length $=315 \mathrm{~cm}$

Span $=205 \mathrm{~cm}$
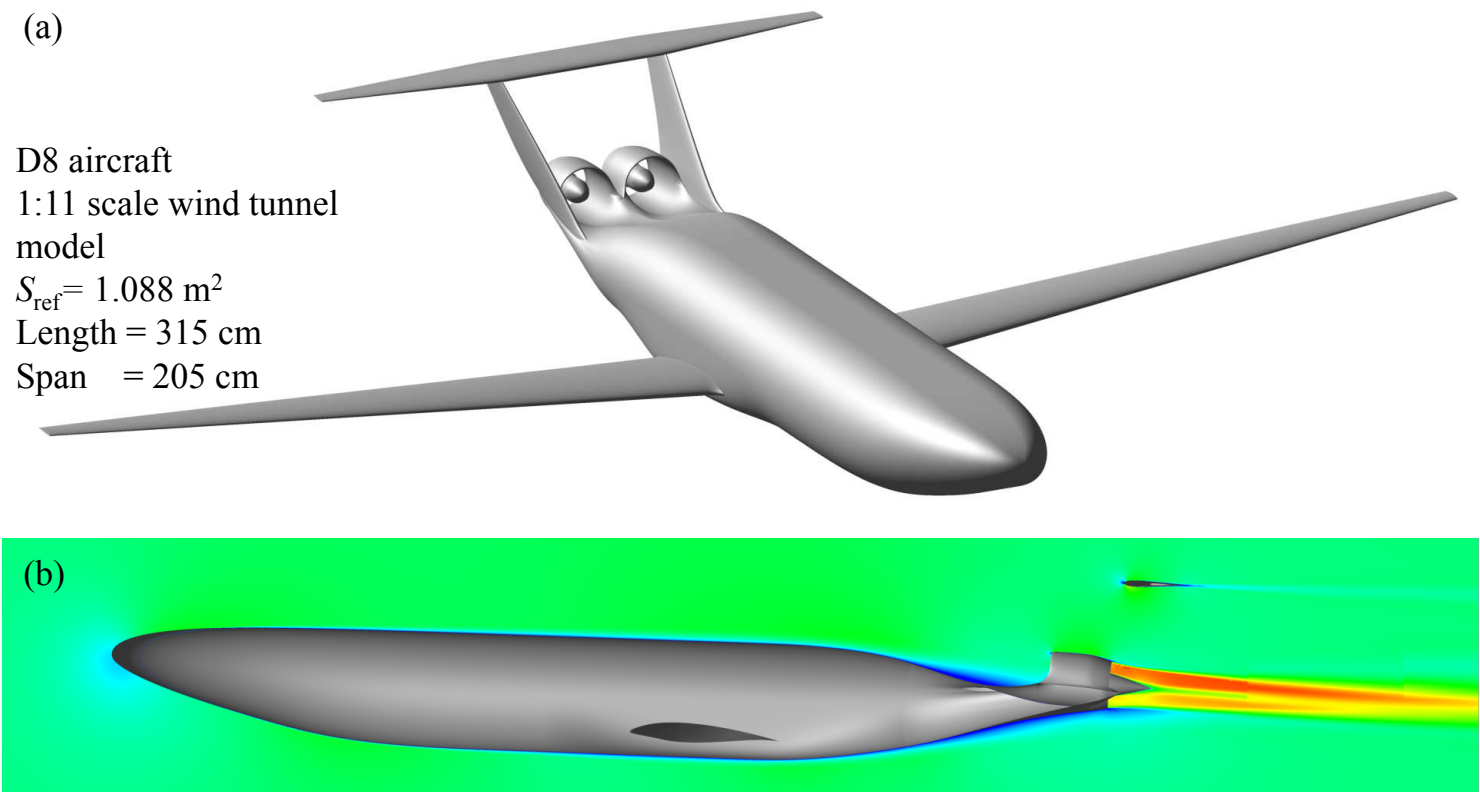

(c)

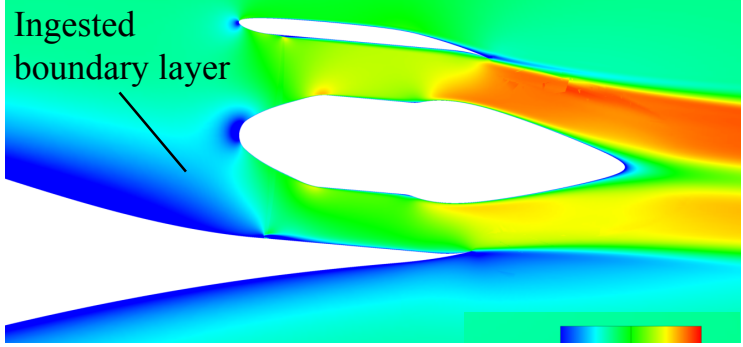

(d)

Figure 1. (a) The overall geometry of the D8 aircraft. (b), (c) The contours of the Mach number and (d) pressure coefficient found by actuator disk boundary condition with uniform pressure jump. 


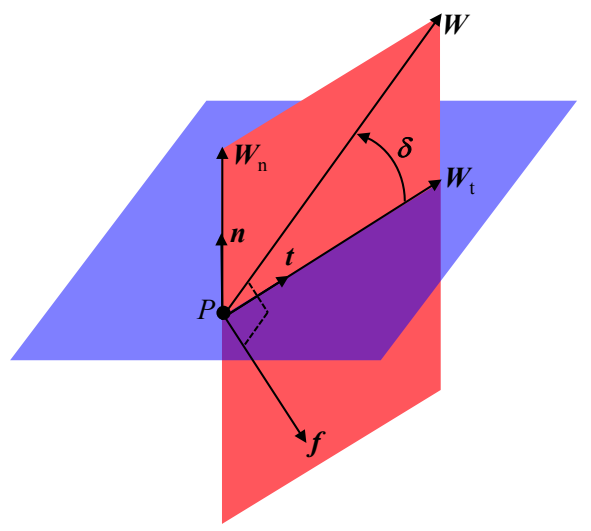

Figure 2. Components of relative velocity $W$ on surfaces tangential and normal to the blade.

(a)

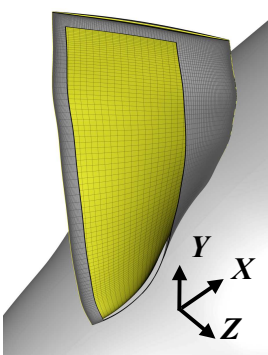

(b)

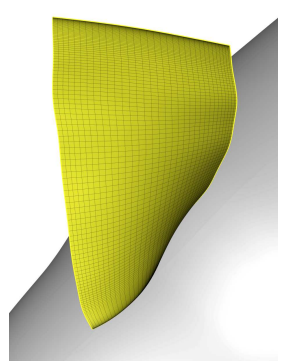

(c)

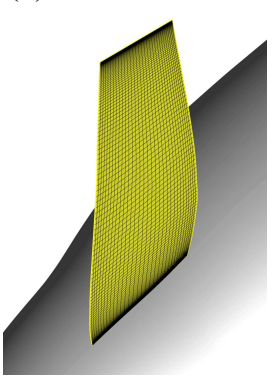

(d)

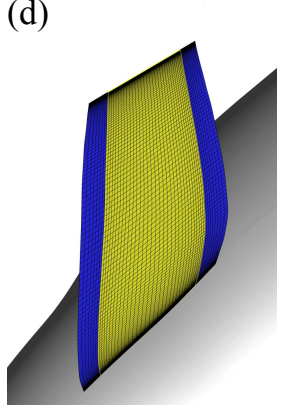

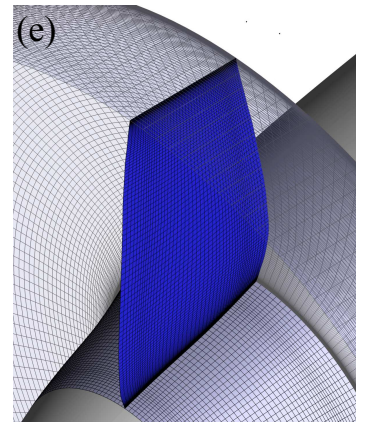

(f)

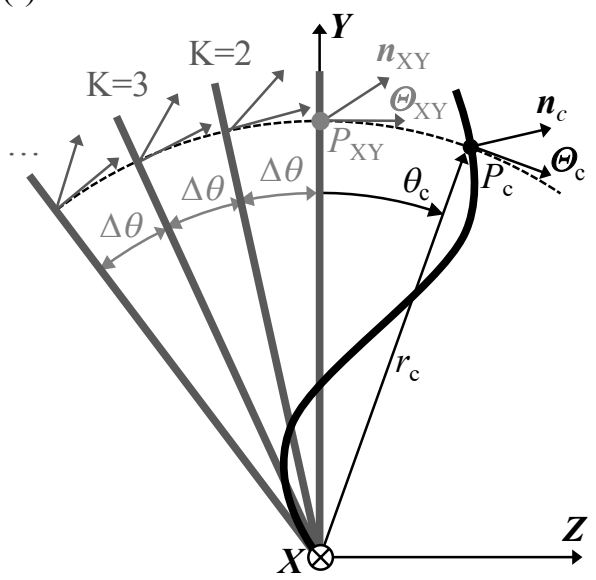

$(\mathrm{g})$

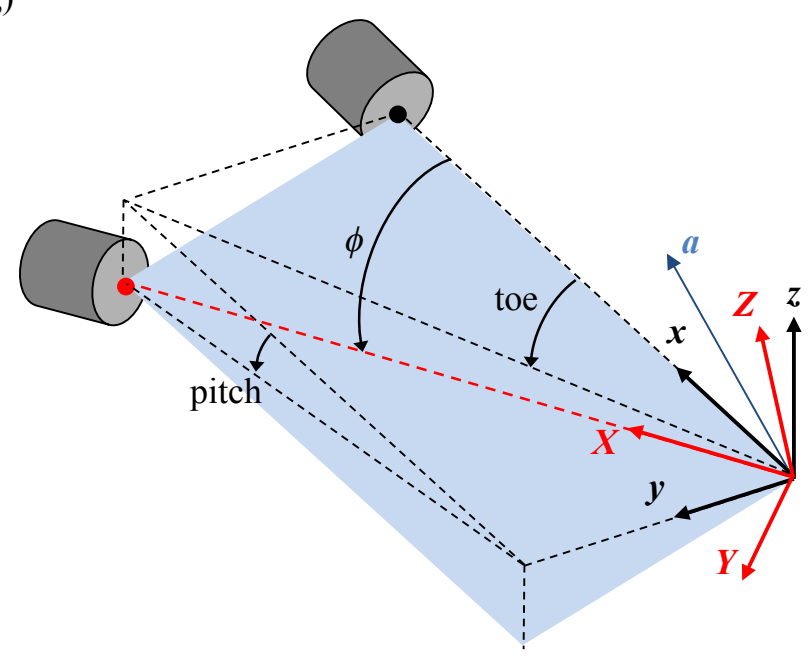

Figure 3. Generation of rotor grid zone for body force model. The same steps apply to the stator grid zone. (a) and (b) Extract the camber surface (yellow) from the blade geometry. Compute the camber surface orientation metrics. (c) and (f) Flatten the camber surface onto $X Y$-plane in a point-matched way. (d) Extend the flattened camber surface a few cells upstream and downstream for proper overlap (blue regions). The body force condition will be applied only on the flattened camber subzone (yellow) of this extended zone. (e) and (f) Revolve the flattened and extended camber surface the $X$-axis $360^{\circ}$ and make a periodic volume gridz one. Reorient the orientation metrics for each periodic plane. (g) If the propulsor has toe and pitch angles with respect to the airframe coordinate system ( $x y z$ ) then rotate the surface orientation metrics as well as the propulsor grids accordingly. 
(a)
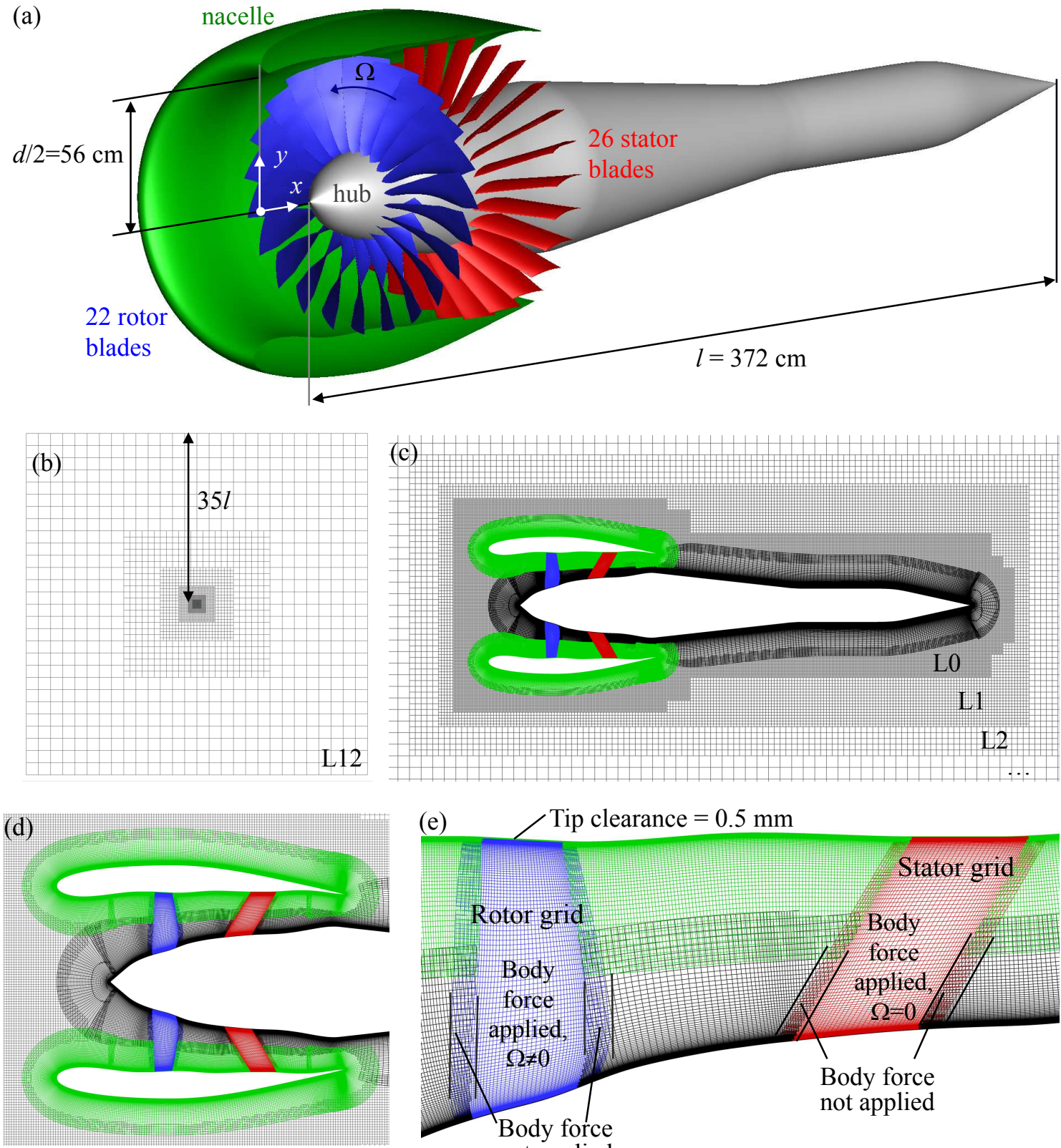

Figure 4. View of SDT fan geometry and the overset grid system in various scopes. (a) The entire fan model (b) Cartesian off-body grids (c) Close up of the model (d) Close up near the nacelle region (e) Close up near the rotor and stator grids 

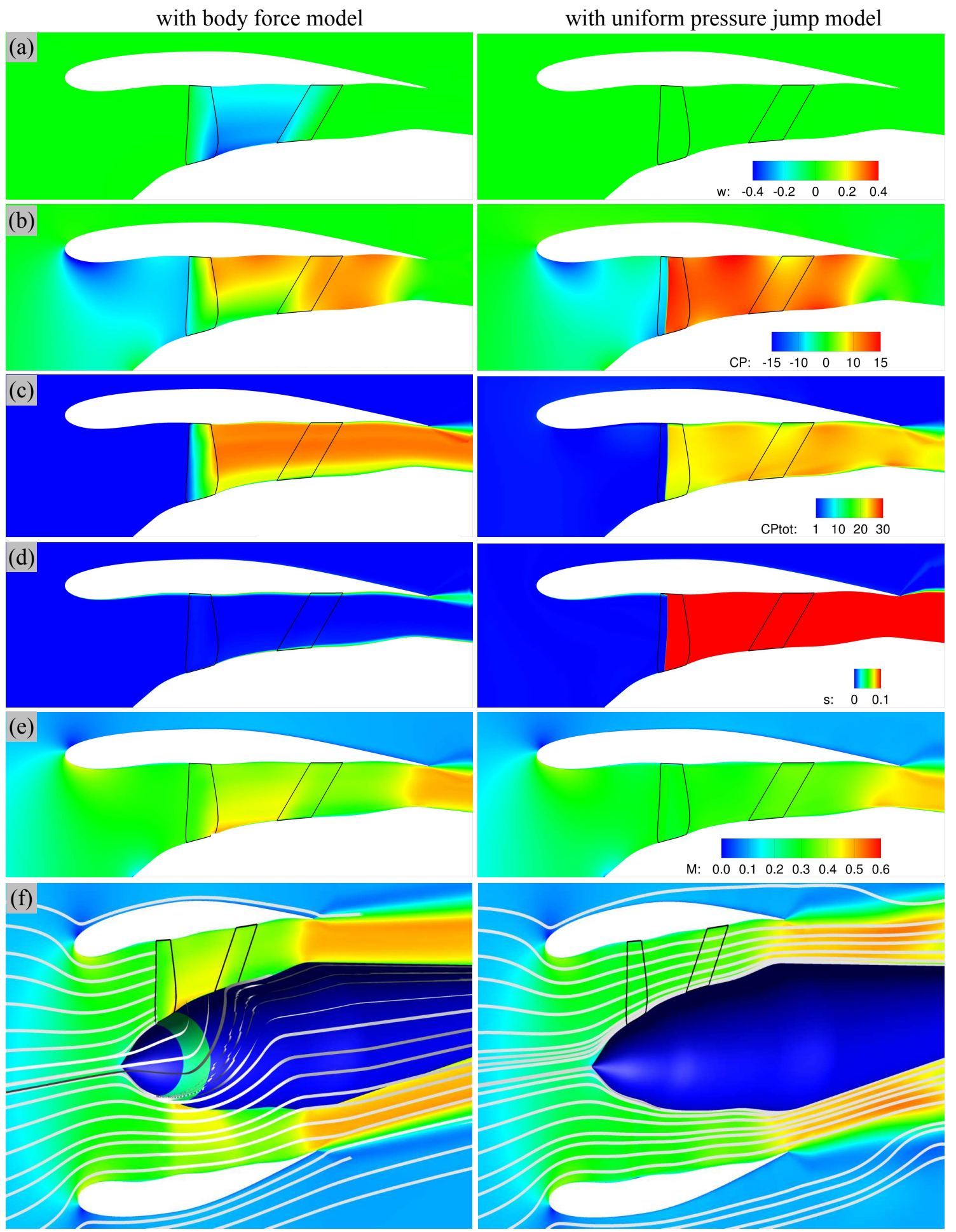

Figure 5. Contour plots on $y=0$ plane of SDT fan. Outlines of the rotor and the stator zones are indicated by solid black lines. On each row (a) to (f), contour plot on left is from body force model and on right from actuator disk model. (a) Out-of-plane component of the local Mach number (b) Pressure coefficient (c) Stagnation pressure coefficient (d) Entropy (e) Mach number (d) Streamlines superposed on the Mach number contours of the same range. View angle is the same as in Figure 4a. Presence of the spinner boundary condition is evident as non-zero Mach number segment on the hub surface. 

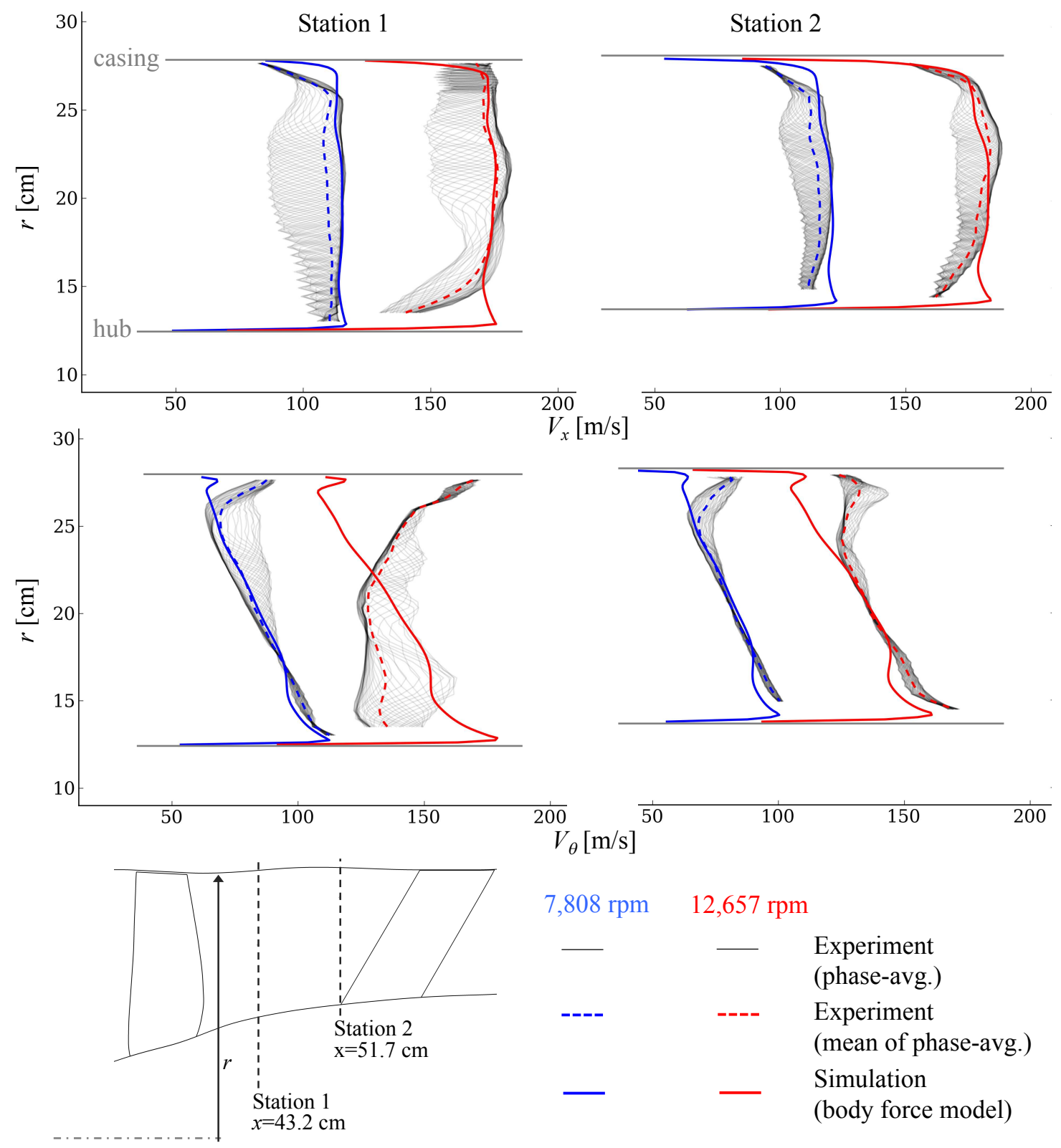

Figure 6. Comparison of streamwise and swirl velocities predicted by the body force model with experimental values obtained by the LDV measurements. 
(a)

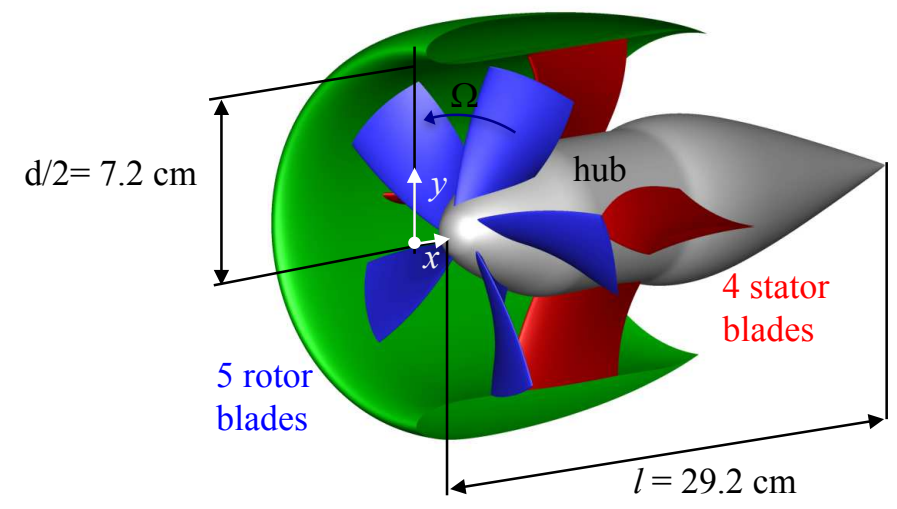

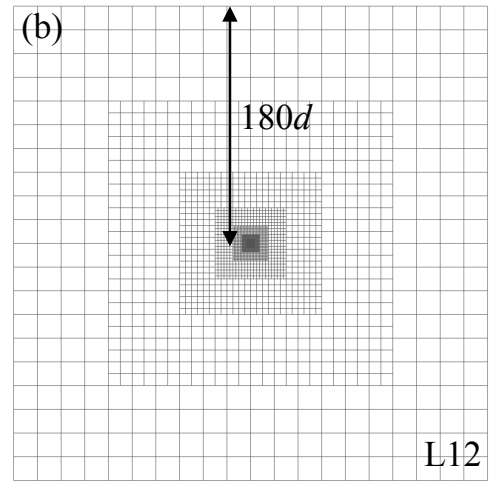

(c)

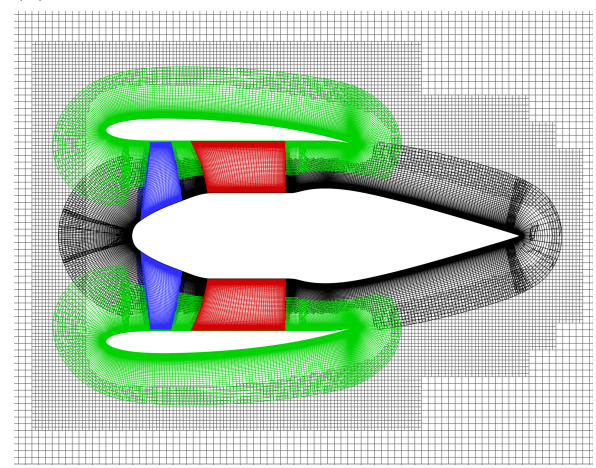

(d)

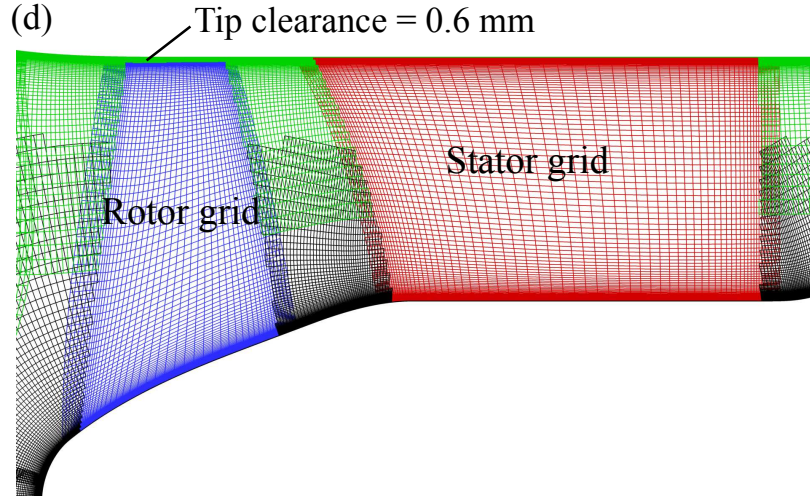

Figure 7. View of TF8000 propulsor geometry and the overset grid system in various scopes. (a) The entire fan model (b) Cartesian off-body grids (c) Close up near the propulsor (d) Close up near the rotor and stator grids 
with body force model
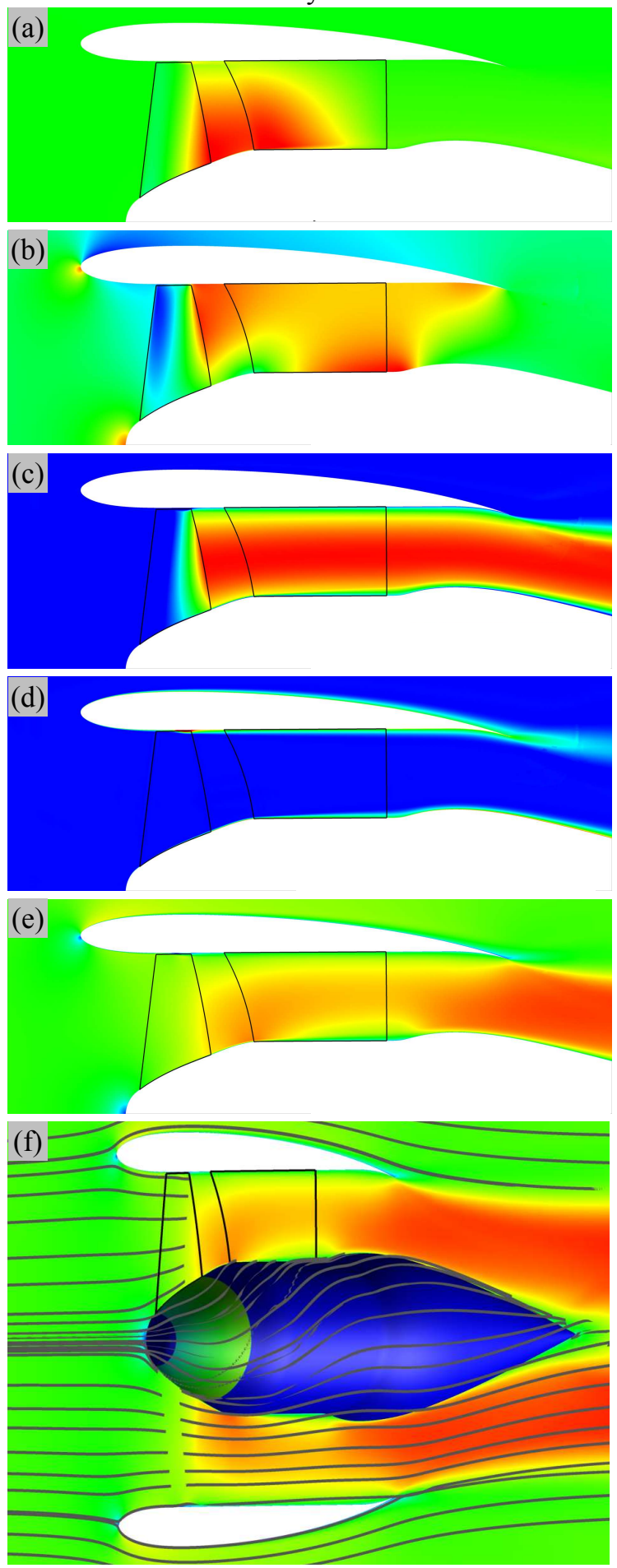

with uniform pressure jump model
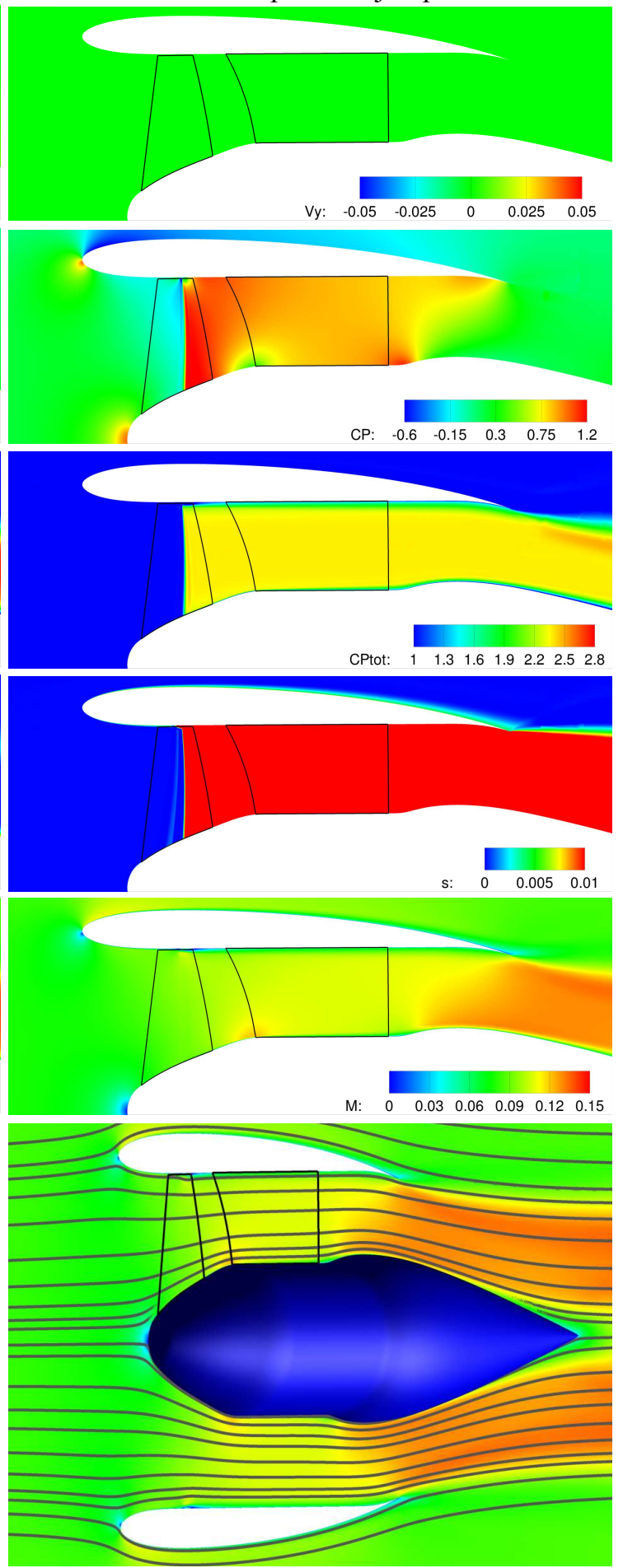

Figure 8. Contour plots on $\mathrm{z}=0$ plane of TF8000 propulsor. Outlines of the rotor and the stator zones are indicated by solid black lines. On each row (a) to (f), the contour plot on the left is from body force model and the one on the is right from the actuator disk model. (a) Out-of-plane component of the local Mach number (b) Pressure coefficient (c) Stagnation pressure coefficient (d) Entropy (e) Mach number (d) Streamlines superposed on the Mach number contours of the same range in a a perspective view. 
with body force model

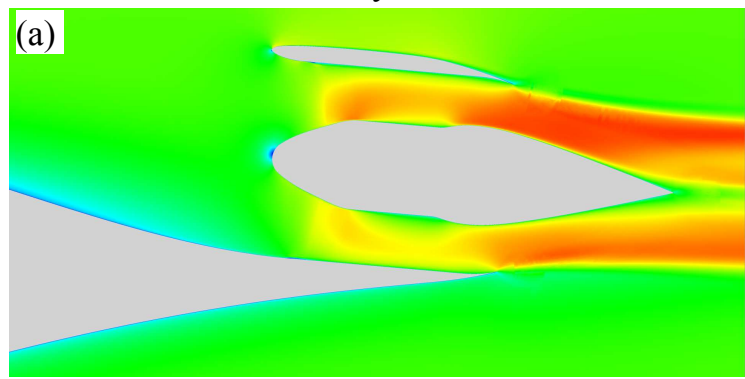

(b)

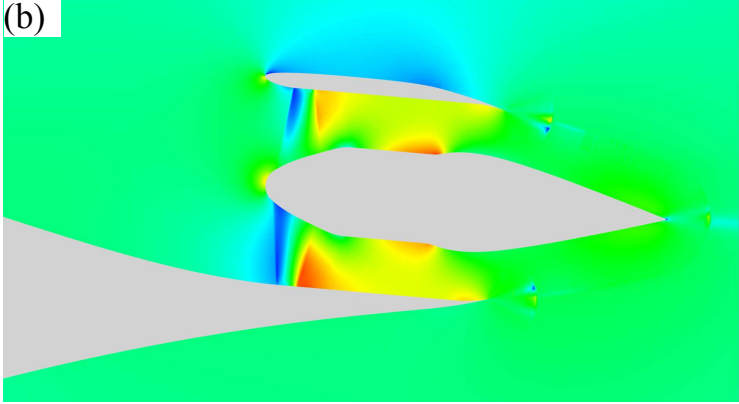

(c)

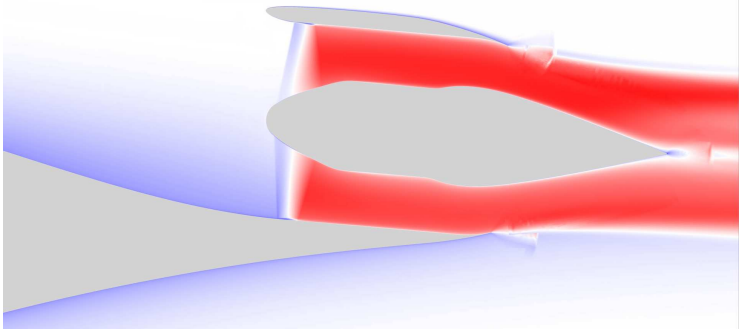

with uniform pressure jump model
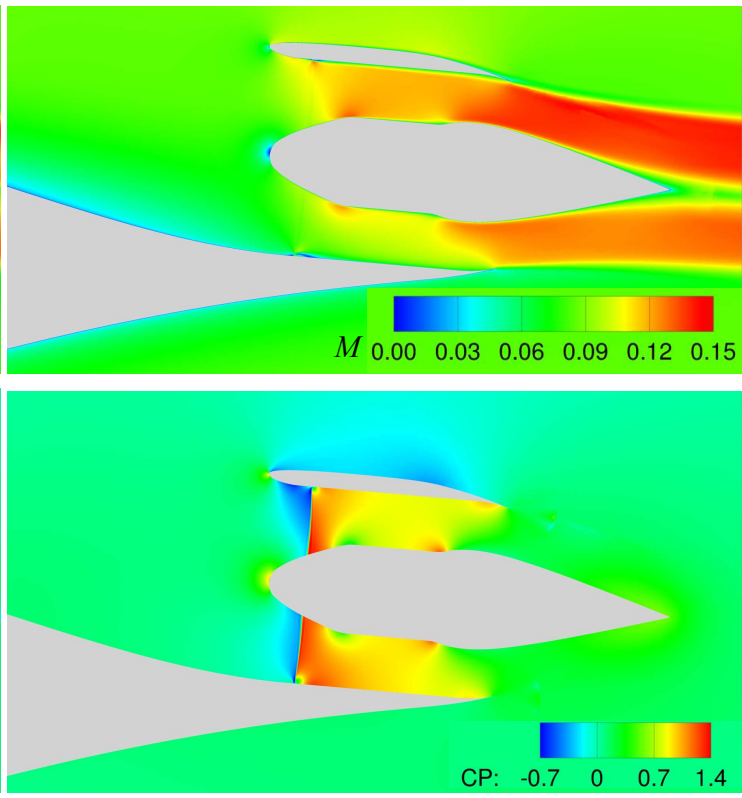

(d)

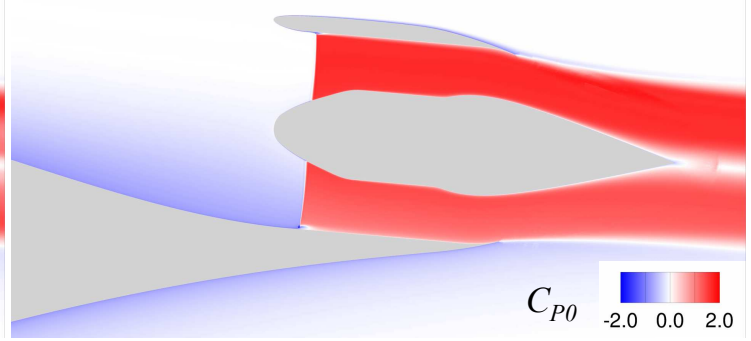

$\Delta C_{P 0}=C_{P 0, \text { body force }}-C_{P 0, \text { pressure jump }}$

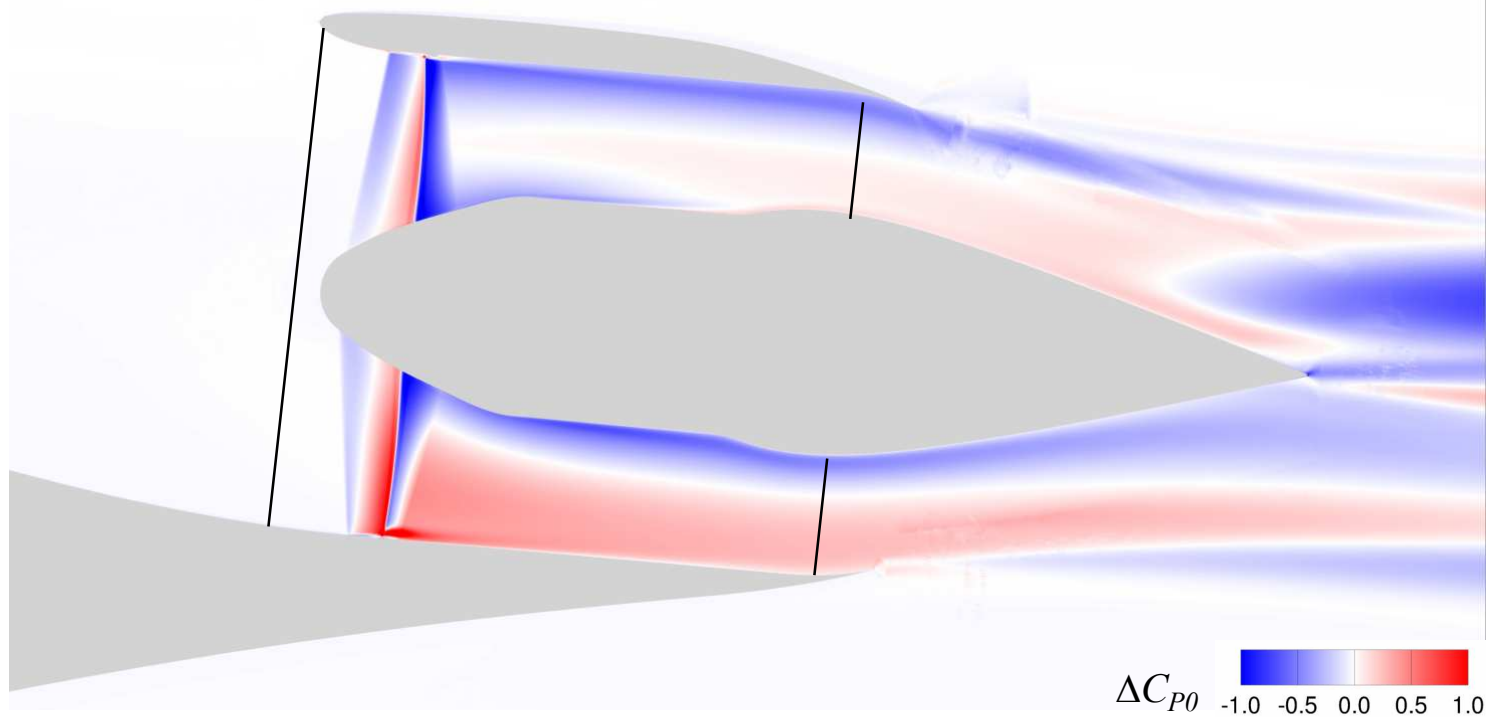

Figure 9. Contour plots on the center plane of TF8000 propulsor installed on D8 Integrated model. (a),(b), (c), Contours of Mach number, pressure coefficient and total pressure coefficient. (d) Difference of the total pressure coefficient fields found by subtracting $C_{P_{0}}$ field found by uniform pressure jump model from that found by body force model. The locations of inlet and exit planes used for $C_{P_{K}}$ integration are indicated by solid plack lines. 
(a) with body force model

with uniform pressure jump model

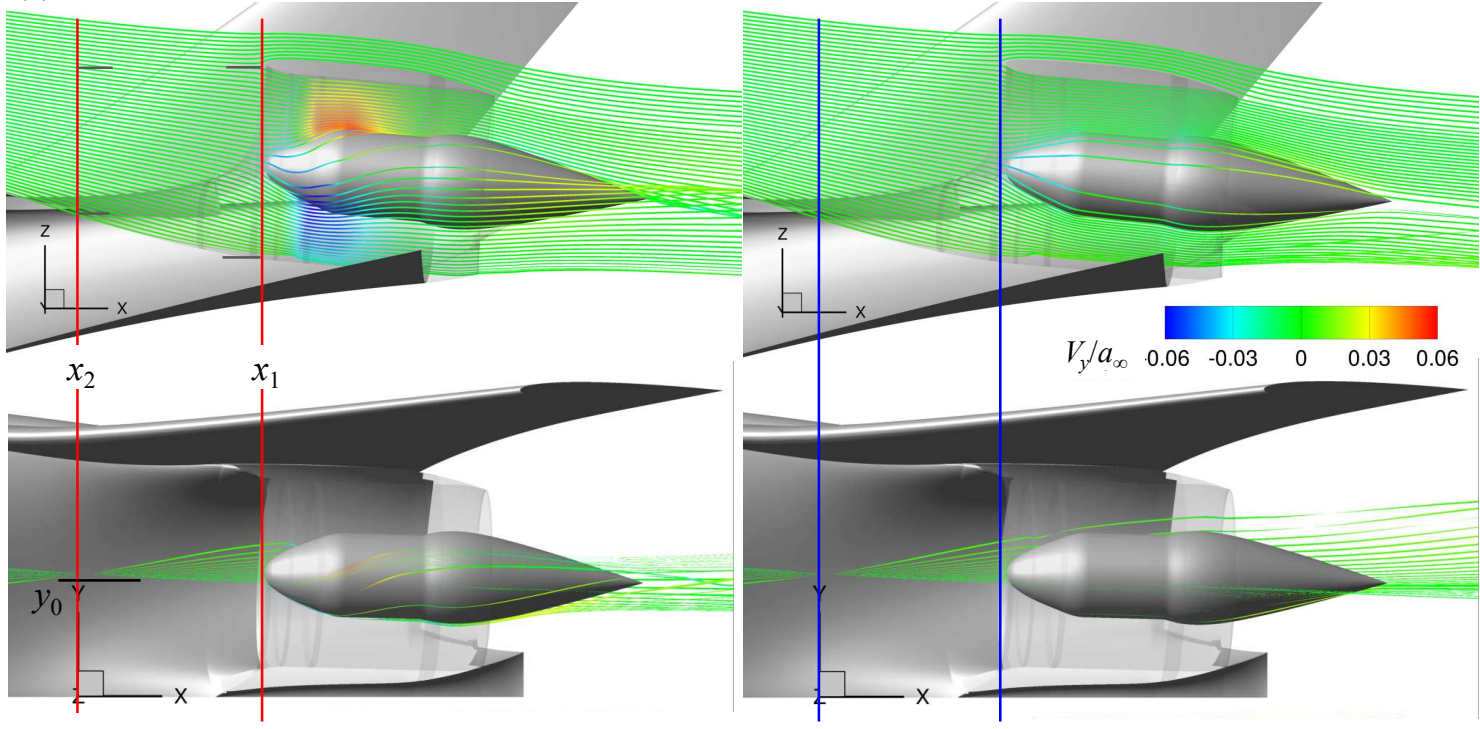

(b) At $y_{0}, x_{1}=2.79 \mathrm{~m}$ :

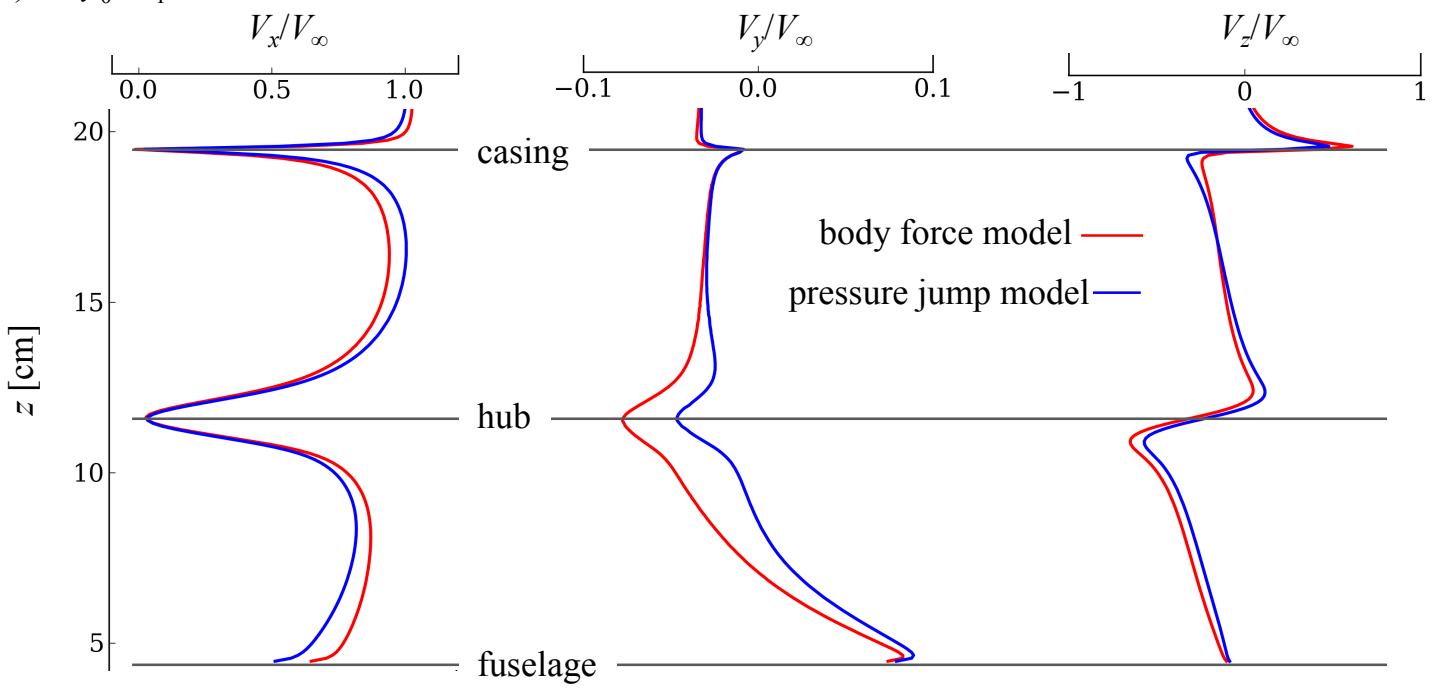

$x_{2}=x_{1}-0.88 d=2.67 \mathrm{~m}:$

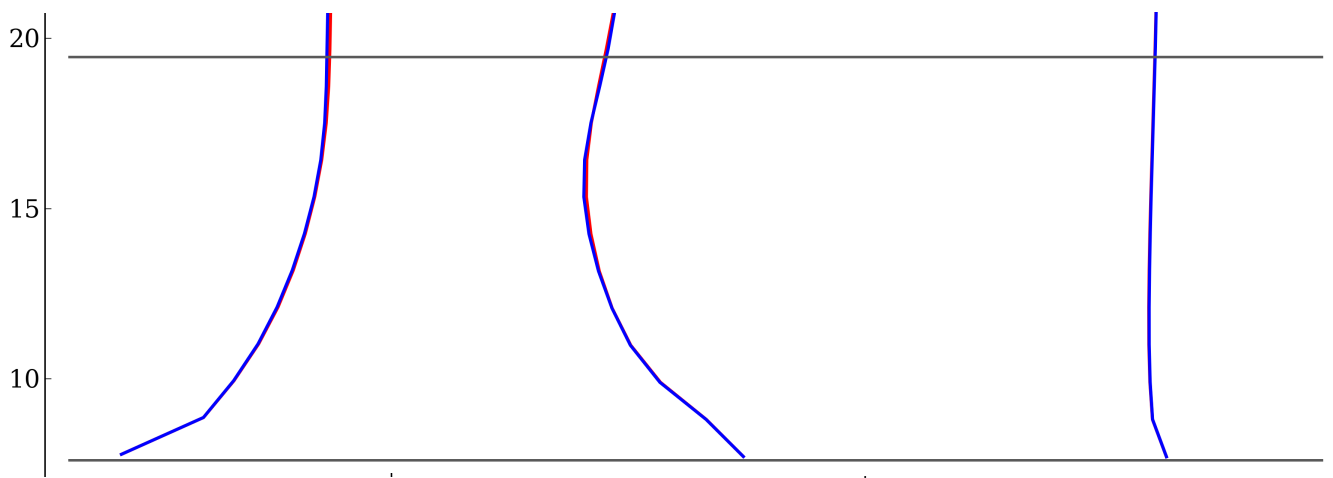

Figure 10. (a) Streamtraces colored by y-velocity normalized by free stream Mach number. Side and top views, respectively, on upper and lower rows. Left column with body force model, right columnt with uniform pressure jump model. (b) Line plots of normalized velocity components along $z$-lines at $\left(x_{1}, y_{0}\right)$ (near fan face) and ( $\left.x_{2}, y_{0}\right)$ (about 1 fan diameter upstream of fan face). Red lines indicate these line data extractions from body force model simulations and blude lines indicate the ones from uniform pressure jump model simulations. 